\title{
Subsystem complexity in warped AdS
}

\author{
Roberto Auzzi, ${ }^{a, b}$ Stefano Baiguera, ${ }^{c}$ Arpita Mitra, ${ }^{e}$ Giuseppe Nardelli ${ }^{a, d}$ \\ and Nicolò Zenoni ${ }^{a, b}$ \\ ${ }^{a}$ Dipartimento di Matematica e Fisica, Università Cattolica del Sacro Cuore, \\ Via Musei 41, 25121 Brescia, Italy \\ ${ }^{b}$ INFN - Sezione di Perugia, \\ Via A. Pascoli, 06123 Perugia, Italy \\ ${ }^{c}$ Università degli studi di Milano Bicocca and INFN — Sezione di Milano-Bicocca, \\ Piazza della Scienza 3, 20161, Milano, Italy \\ dTIFPA - INFN, c/o Dipartimento di Fisica, Università di Trento, \\ 38123 Povo TN, Italy \\ ${ }^{e}$ Indian Institute of Science Education and Research Bhopal, \\ Bhopal Bypass, Bhauri, Bhopal 462006, India \\ E-mail: roberto.auzzi@unicatt.it, s.baiguera@campus.unimib.it, \\ arpitam@iiserb.ac.in, giuseppe.nardelli@unicatt.it, \\ nicolo.zenoni@unicatt.it
}

ABSTRACT: We compute the ultraviolet divergences of holographic subregion complexity for the left and right factors of the thermofield double state in warped $\mathrm{AdS}_{3}$ black holes, both for the action and the volume conjectures. Besides the linear divergences, which are also present in the BTZ black hole, additional logarithmic divergences appear. For the action conjecture, these log divergences are not affected by the arbitrarity in the length scale associated with the counterterm needed to ensure reparameterization invariance. We find that the subregion action complexity obeys the superadditivity property for the thermofield double in warped $\mathrm{AdS}_{3}$, independently from the action counterterm coefficient. We study the temperature dependence of subregion complexity at constant angular momentum and we find that it is correlated with the sign of the specific heat.

KeYwords: AdS-CFT Correspondence, Black Holes

ARXIV EPRINT: 1906.09345 


\section{Contents}

1 Introduction 1

2 Black holes in warped $\mathrm{AdS}_{3}$ spacetime $\quad 4$

2.1 An explicit realization in Einstein gravity 5

$\begin{array}{ll}2.2 & \text { Eddington-Finkelstein coordinates }\end{array}$

$\begin{array}{lll}3 & \text { Action } & 7\end{array}$

$\begin{array}{lll}3.1 & \text { Contributions to the action } & 7\end{array}$

$\begin{array}{ll}3.2 \text { Total action } & 10\end{array}$

$\begin{array}{ll}3.3 & \text { Action of internal region and subregion complexity } \\ \end{array}$

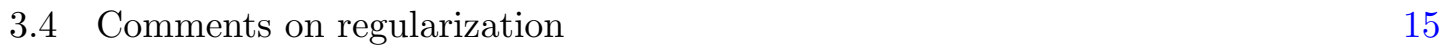

$\begin{array}{ll}3.5 & \text { Comments on counterterms } \\ \end{array}$

$\begin{array}{llr}4 & \text { Volume } & 17\end{array}$

$\begin{array}{lll}4.1 & \text { CV conjecture } & 17\end{array}$

$\begin{array}{ll}4.2 & \text { Spacetime volume (CV 2.0) } \\ \end{array}$

$\begin{array}{llr}5 & \text { Conclusions } & 18\end{array}$

A Non-rotating case $\quad 21$

$\begin{array}{lll}\text { A.1 Total action } & 21\end{array}$

$\begin{array}{lll}\text { A.2 External action } & 22\end{array}$

A.3 Volume 23

B Subsystem complexity and temperature $\quad 24$

\section{Introduction}

According to the AdS/CFT correspondence, eternal black holes in AdS are dual to the thermofield double state $[1,2]$, which corresponds to two copies of entangled conformal field theories living on the left and right boundaries of the Penrose diagram. Quantum information concepts such as entanglement entropy have the potential to give us essential information on how spacetime can emerge from the boundary field theory in holographic dualities.

Recently, the new concept of computational complexity has been introduced in order to provide a field theory dual to the linear growth of the Einstein-Rosen Bridge inside the horizon of a Black Hole (BH) [3-5]. The concept of complexity originates in theoretical computer science. In quantum computing, it is defined as the minimal number of elementary unitary operations which are needed in order to prepare a given state from a reference state. In quantum mechanics, this is a function of the chosen reference state, of the set of 
of quantum gates and of the allowed tolerance in the accuracy with which the final state is prepared. An elegant geometric approach to complexity was developed by Nielsen and collaborators $[6,7]$. In conformal field theories (CFTs), a precise definition of complexity is still lacking. Some possible definitions for free field theories have been studied by several authors, e.g. [8-14]. Another approach for 2-dimensional field theories uses the Liouville action $[15,16]$. This research field is still in its nascent stages.

There are a few proposals for the gravitational dual of complexity in the AdS/CFT correspondence:

- the Complexity=Volume $(\mathrm{CV})[3-5]$ refers to the spacetime volume $V$ of an extremal spacelike codimension-1 slice anchored at the boundary. We will denote the corresponding complexity as $\mathcal{C}_{V}$ :

$$
\mathcal{C}_{V}=\operatorname{Max}\left(\frac{V}{G l}\right)
$$

where $G$ is the gravitational constant and $l$ the AdS radius.

- the Complexity=Action (CA) $[17,18]$ refers to a gravitational action $I_{\text {WDW }}$ computed in the Wheeler-De Witt (WDW) patch, which is the union of all the spacelike slices that can be attached to the boundary at a given time. The gravitational action must include also a set of boundary terms needed to consistently define an additive and a reparameterization-invariant bulk action. This includes the Gibbons-Hawking-York term, the terms due to the presence of null boundaries and their intersections $[19,20]$. We denote the action complexity as $\mathcal{C}_{A}$ :

$$
\mathcal{C}_{A}=\frac{I_{\mathrm{WDW}}}{\pi} .
$$

- the Complexity= Spacetime Volume (CV 2.0) [21] refers to the spacetime volume of the WDW patch:

$$
\mathcal{C}_{V}^{2.0} \propto V_{\mathrm{WDW}}
$$

These proposals share several common qualitative behaviours. Our understanding of complexity in field theory is not good enough at the moment to precisely discriminate among them. CA appears as more universal, because the CV proposal requires the introduction of an ad hoc length scale to relate complexity, which is dimensionless, to a spacetime volume, which is a dimensional quantity. On the other hand, CV shows a more regular monotonically increasing growth rate for intermediate times [22], which matches expectations from quantum circuits. See e.g. [23-35] for several recent investigations on the topic of holographic complexity.

By analogy with entanglement entropy, an interesting further extension of the $\mathrm{CV}$ and CA conjectures is to restrict to subregions of the full boundary theory. This should be dual to some notion of subsystem complexity of a mixed state on the boundary. The most promising bulk region which should correspond to a boundary density matrix localised in a subregion is the entanglement wedge [36], and so it is natural to propose that subregion complexity is dual to quantities which have support in the entanglement wedge. For CV, it 
was conjectured [37] that mixed state complexity is dual to the volume of the codimension-1 extremal slice in the bulk attached to the boundary subregion and its Ryu-Takayanagi (RT) [38] surface. In the CA framework, it was proposed in [39] that the mixed state complexity is dual to the action of the intersection of the WDW patch and the entanglement wedge associated to the given spatial subregion. Other works on holographic subregion complexity include [40-45].

There are a few different possible definitions of complexity of a mixed state $\rho$ localised in a subregion of the Hilbert space of a quantum field theory [46]:

- purification complexity $\mathcal{C}_{P}$, which can be defined as the minimal number of gates needed to transform the initial pure state (plus some ancillary external qubits) into a purification of the mixed state $\rho$;

- spectrum complexity $\mathcal{C}_{S}$, which can be defined as the minimal number of operations needed to prepare a mixed state $\rho_{\text {spec }}$ with the same spectrum as $\rho$;

- basis complexity $\mathcal{C}_{B}$, which can be defined as the minimum number of gates needed to prepare $\rho$ from $\rho_{\text {spec }}$.

The spectrum complexity does not reduce to complexity when computed on pure states, and so it is not a good candidate as a field theory dual of CV or CA. Instead both $\mathcal{C}_{P}$ and $\mathcal{C}_{B}$ might be in principle reasonable candidates as duals of holographic complexities. These issues were recently investigated by [46-49].

In particular, it was conjectured [46] that $\mathcal{C}_{P}$ should be subadditive for the left $L$ and right $R$ factors of the thermofield double state $T D$. An analog guess was made about superadditivity of $\mathcal{C}_{B}$. If these conjectures were true, they would be useful to discriminate which notion of subregion complexity is dual to a given holographic realisation.

The volume complexity $\mathcal{C}_{V}$ is in general superadditive because the volume is always a positive-definite quantity:

$$
\mathcal{C}_{V}(A B) \geq \mathcal{C}_{V}(A)+\mathcal{C}_{V}(B) .
$$

Moreover, for the thermofield double at time zero, this inequality saturates:

$$
\mathcal{C}_{V}(T D, t=0)=\mathcal{C}_{V}(L)+\mathcal{C}_{V}(R) .
$$

For $\mathcal{C}_{A}$ the situation is murky, because action is not positive-definite. An interesting technical point which arises in the CA conjecture is due to an arbitrary length scale $\tilde{L}$ which appears in a counterterm which is needed in order to make the action reparameterization invariant [20]. Depending on the choice of $\tilde{L}$, for the AdS neutral black hole, one can get either [47] that $\mathrm{CA}$ is superadditive or subadditive for the $L, R$ sides of the thermofield double.

Another interesting property is the temperature behaviour of mixed state complexity. In [46] it was argued from tensor network arguments that $\mathcal{C}_{B}$ decreases with temperature $T$ and approaches zero for $T \rightarrow \infty$, while $\mathcal{C}_{P}$ should not have strong dependence on $T$. As studied in $[46,47]$, for the AdS neutral black hole the behaviour of subsystem action complexity as a function of temperature also depends on $\tilde{L}$. 
An interesting ultraviolet modification of the AdS/CFT correspondence in which many results of holography can be generalised and extended is the Warped $\mathrm{AdS}_{3} / \mathrm{WCFT}_{2}$ correspondence [50-54]. This is a correspondence between gravitational bulk theories in $2+1$ dimensions in a spacetime with Warped $\mathrm{AdS}_{3}\left(\mathrm{WAdS}_{3}\right)$ asymptotic metric and a class of non-relativistic theories in $1+1$ dimensions on the boundary. These are called Warped Conformal Field Theories (WCFTs), and are invariant under the Virasoro and the U(1) Kac-Moody current algebras. They provide a natural direction to extend holography in a non-relativistic direction. For example, a Cardy formula reproducing the black hole entropy was derived in [52]. Entanglement entropy has been studied by several authors [55-59]. Complexity was investigated in [60-63].

In this work we compute the divergences of subregion complexity for the left and right factors of the thermofield double state, in the case of black holes in asymptotically $\mathrm{WAdS}_{3}$ spacetimes. We investigate the temperature dependence of subregion complexity in each of the conjectures and the sub/superadditivity properties of the CA conjecture. These properties may help to discriminate which notion of subregion complexity (for example $\mathcal{C}_{P}$ or $\mathcal{C}_{B}$ ) is dual to each of the holographic complexity conjectures.

We find several features that differ from the AdS case:

- the structure of divergences of subregion complexity is different from the AdS case: besides the linear term in the cutoff $\Lambda$, an additional $\log \Lambda$ divergence arises.

- subregion $\mathcal{C}_{A}$ is always superadditive for the $L, R$ factors of TD. Instead in AdS the sub/superadditivity property is dependent on the arbitrary parameter $\tilde{L}$.

- subregion complexities have a temperature dependence which is correlated with specific heat.

The paper is organized as follows: in section 2 we review some basic properties of warped black holes realised in Einstein gravity. In section 3 we compute the divergences of total and subregion action for rotating black holes. In section 4 we compute these divergences in CV and CV 2.0. We conclude and discuss our results in section 5. The details of the calculation for the non-rotating case and some other technical details are deferred to the appendices.

\section{Black holes in warped $\mathrm{AdS}_{3}$ spacetime}

Black holes in asymptotically warped $\mathrm{AdS}_{3}$ spacetime $[50,64,65]$ are described by the following metric

$$
\frac{d s^{2}}{l^{2}}=d t^{2}+\frac{d r^{2}}{\left(\nu^{2}+3\right)\left(r-r_{+}\right)\left(r-r_{-}\right)}+\left(2 \nu r-\sqrt{r_{+} r_{-}\left(\nu^{2}+3\right)}\right) d t d \theta+\frac{r}{4} \Psi(r) d \theta^{2}
$$

where

$$
\Psi(r)=3\left(\nu^{2}-1\right) r+\left(\nu^{2}+3\right)\left(r_{+}+r_{-}\right)-4 \nu \sqrt{r_{+} r_{-}\left(\nu^{2}+3\right)},
$$


the inner and outer horizons are placed in $r_{-}, r_{+}$and $\nu$ is a warping parameter such that for $\nu=1$ the metric gives the BTZ black hole $[66,67]$. We define $\rho_{0}$ as the zero of the function $\Psi(r)$, i.e.:

$$
\rho_{0}=\frac{4 \nu \sqrt{r_{+} r_{-}\left(\nu^{2}+3\right)}-\left(\nu^{2}+3\right)\left(r_{+}+r_{-}\right)}{3\left(\nu^{2}-1\right)} .
$$

We introduce $\tilde{r}_{0}$, defined by

$$
\tilde{r}_{0}=\max \left(0, \rho_{0}\right) \text {, }
$$

so that the range of variables is: $\tilde{r}_{0} \leq r<\infty,-\infty<t<\infty$ and $\theta \sim \theta+2 \pi$.

This metric is pathologic when $\nu^{2}<1$, because admits closed timelike curves. Temperature and angular velocity of the outer horizon are [50]:

$$
T=\frac{\nu^{2}+3}{4 \pi l} \frac{r_{+}-r_{-}}{2 \nu r_{+}-\sqrt{\left(\nu^{2}+3\right) r_{+} r_{-}}}, \quad \Omega=\frac{2}{\left(2 \nu r_{+}-\sqrt{\left.\left(\nu^{2}+3\right) r_{+} r_{-}\right)} l\right.} .
$$

Entropy, mass and angular momentum depend on the gravitational action we choose, and for our computations we will consider warped BHs arising as a solution of Einstein gravity plus Maxwell and Chern-Simons terms [68, 69]. The entropy is then proportional to the area of the event horizon

$$
S=\frac{l \pi}{4 G}\left(2 \nu r_{+}-\sqrt{r_{+} r_{-}\left(\nu^{2}+3\right)}\right),
$$

and the conserved charges (mass and angular momentum) are [68, 69]:

$$
\begin{aligned}
M & =\frac{1}{16 G}\left(\nu^{2}+3\right)\left(\left(r_{-}+r_{+}\right)-\frac{\sqrt{r_{+} r_{-}\left(\nu^{2}+3\right)}}{\nu}\right), \\
J & =\frac{l}{32 G}\left(\nu^{2}+3\right)\left(\frac{r_{-} r_{+}\left(3+5 \nu^{2}\right)}{2 \nu}-\left(r_{+}+r_{-}\right) \sqrt{\left(3+\nu^{2}\right) r_{+} r_{-}}\right) .
\end{aligned}
$$

\subsection{An explicit realization in Einstein gravity}

The solution in eq. (2.1) can be obtained as a vacuum solution of Topological Massive Gravity [50,64, 65] or New Massive Gravity [70]. We will instead focus on realizations of the metric (2.1) in Einstein gravity. Unfortunately, all the known explicit constructions of $\mathrm{WAdS}_{3}$ black holes in Einstein gravity have some pathology in the matter content. For concreteness we will use a model introduced in [68], where the matter content is a ChernSimons U(1) gauge field. In order to find absence of closed timelike curves $\left(\nu^{2} \geq 1\right)$, a ghost-like kinetic Maxwell term is needed. This is the same theoretical setting that was studied in [62], where we found that the asymptotic growth of $\mathcal{C}_{A}$ was, as expected, proportional to $T S$. This is also consistent with the CV conjecture [61]. The CA conjecture seems solid enough to survive to unphysical matter contents which include ghosts.

We consider Einstein gravity in 3 dimensions with a negative cosmological constant, coupled to a U(1) gauge field with both Maxwell and Chern-Simons terms [68]:

$$
I_{\mathcal{V}}=\frac{1}{16 \pi G} \int_{\mathcal{V}} d^{3} x\left\{\sqrt{-g}\left[\left(R+\frac{2}{L^{2}}\right)-\frac{\kappa}{4} F_{\mu \nu} F^{\mu \nu}\right]-\frac{\alpha}{2} \epsilon^{\mu \nu \rho} A_{\mu} F_{\nu \rho}\right\}=\int_{\mathcal{V}} d^{3} x \sqrt{-g} \mathcal{L}
$$


We use the same notation as in [62]. The following solution for the gauge field is considered:

$$
A=a d t+\operatorname{crd\theta }, \quad F=c d r \wedge d \theta
$$

The Maxwell and Einstein equations give:

$$
\alpha=\kappa \frac{\nu}{l}, \quad L=l \sqrt{\frac{2}{3-\nu^{2}}}, \quad c= \pm l \sqrt{\frac{3}{2} \frac{1-\nu^{2}}{\kappa}} .
$$

In order to avoid closed timelike curves, we have to impose $\nu \geq 1$, which implies the ghost condition $\kappa<0$.

The gauge parameter $a$ is not constrained by the equations of motion, but the action depends explicitly on $a$ through the Chern-Simons term. The value of $a$ is important to properly define the mass $M$ as a conserved charge [69]. Formally, only for the value

$$
a=\frac{l}{\nu} \sqrt{\frac{3}{2}} \sqrt{\nu^{2}-1}
$$

the mass is associated to the Killing vector $\partial / \partial t$ and it does not depend on the $\mathrm{U}(1)$ gauge transformations. For this value, the action density reads:

$$
16 \pi G \sqrt{-g} \mathcal{L}=-\frac{l}{2}\left(\nu^{2}+3\right)=\mathcal{I} .
$$

\subsection{Eddington-Finkelstein coordinates}

It is convenient to introduce null coordinates using the ADM decomposition of the metric $(2.1)$ :

$$
d s^{2}=-N^{2} d t^{2}+\frac{l^{4} d r^{2}}{4 R^{2} N^{2}}+l^{2} R^{2}\left(d \theta+N^{\theta} d t\right)^{2}
$$

where

$$
R^{2}=\frac{r}{4} \Psi, \quad N^{2}=\frac{l^{2}\left(\nu^{2}+3\right)\left(r-r_{+}\right)\left(r-r_{-}\right)}{4 R^{2}}, \quad N^{\theta}=\frac{2 \nu r-\sqrt{r_{+} r_{-}\left(\nu^{2}+3\right)}}{2 R^{2}} .
$$

In order to delimit the WDW patch, we use the set of null coordinates introduced in [71], obtained by considering a set of null geodesics which satisfies $\left(d \theta+N^{\theta} d t\right)=0$. Then the last term in the metric (2.14) saturates to zero, and the null geodesics are parameterized by the constant $u$ and $v$ trajectories:

$$
d u=u_{\alpha} d x^{\alpha}=d t-\frac{l^{2}}{2 R N^{2}} d r, \quad d v=v_{\alpha} d x^{\alpha}=d t+\frac{l^{2}}{2 R N^{2}} d r .
$$

These one-forms are both normal and tangent to the null boundaries of the WDW patch. Moreover, the integral curves of $u^{\alpha}$ and $v^{\alpha}$ are null geodesics in the affine parameterization, i.e.

$$
u^{\alpha} D_{\alpha} u^{\beta}=0, \quad v^{\alpha} D_{\alpha} v^{\beta}=0,
$$

where $D_{\alpha}$ is the covariant derivative. 
From eq. (2.16), we find the null coordinates

$$
u=t-r^{*}(r), \quad v=t+r^{*}(r),
$$

where the tortoise coordinate $r^{*}$ is given by

$$
r^{*}(r)=\int^{r} \frac{d r^{\prime}}{f\left(r^{\prime}\right)}, \quad f(r)=\frac{2 R N^{2}}{l^{2}}=\frac{\left(\nu^{2}+3\right)\left(r-r_{-}\right)\left(r-r_{+}\right)}{\sqrt{r \Psi(r)}} .
$$

Integrating eq. (2.19), $r^{*}$ can be explicitly found [71]; for $r_{+} \neq r_{-}$the explicit expression is

$$
\begin{aligned}
r^{*}(r)= & \frac{\sqrt{3\left(\nu^{2}-1\right)}}{\left(\nu^{2}+3\right)}\left\{\frac{\sqrt{r_{+}\left(r_{+}-\rho_{0}\right)}}{r_{+}-r_{-}} \log \left(\frac{\left|r-r_{+}\right|}{\left(\sqrt{r} \sqrt{r_{+}-\rho_{0}}+\sqrt{r-\rho_{0}} \sqrt{r_{+}}\right)^{2}}\right)\right. \\
& \left.-\frac{\sqrt{r_{-}\left(r_{-}-\rho_{0}\right)}}{r_{+}-r_{-}} \log \left(\frac{\left|r-r_{-}\right|}{\left(\sqrt{r} \sqrt{r_{-}-\rho_{0}}+\sqrt{r-\rho_{0}} \sqrt{r_{-}}\right)^{2}}\right)+2 \log \left(\sqrt{r}+\sqrt{r-\rho_{0}}\right)\right\} .
\end{aligned}
$$

The tortoise coordinate $r^{*}$ is divergent at $r \rightarrow \infty$, with leading behaviour

$$
\lim _{r \rightarrow \infty} r^{*}(r) \approx \frac{\sqrt{3\left(\nu^{2}-1\right)}}{\nu^{2}+3} \log r
$$

The non-rotating case is defined by $J=0$, and is realised by one of the following conditions:

$$
r_{-}=0, \quad \frac{r_{+}}{r_{-}}=\frac{4 \nu^{2}}{\nu^{2}+3} .
$$

The corresponding Penrose diagram is the same as the one for Schwarzschild BH in four dimensions [71]. The two values in eq. (2.22) can be mapped one into the other by an isometry [71], and so for simplicity we will always consider the case $r_{-}=0, r_{+}=r_{h}$ when we will refer to the non-rotating case.

For generic values $\left(r_{+}, r_{-}\right)$we get a rotating $\mathrm{BH}$, and the Penrose diagram is the same as the one of the Reissner-Nordström BH. The extremal limit corresponds to $r_{+}=r_{-}$; in this case the temperature is zero and there is no thermofield double: the Penrose diagram has just one boundary. We are not interested in this case in the present paper.

\section{Action}

\subsection{Contributions to the action}

The action of the WDW patch has several contributions, which can be evaluated using the results of [20]:

$$
I=I_{\mathcal{V}}+I_{\mathcal{B}}+I_{\mathcal{J}}+I_{\mathrm{ct}}
$$

Here $I_{\mathcal{V}}$ refers to the bulk, $I_{\mathcal{B}}$ to the codimension- 1 boundaries and $I_{\mathcal{J}}$ to the codimension-2 joints coming from intersections of other boundaries. The contribution $I_{\mathrm{ct}}$ is a counterterm to be added in order to ensure reparameterization invariance of the action. 

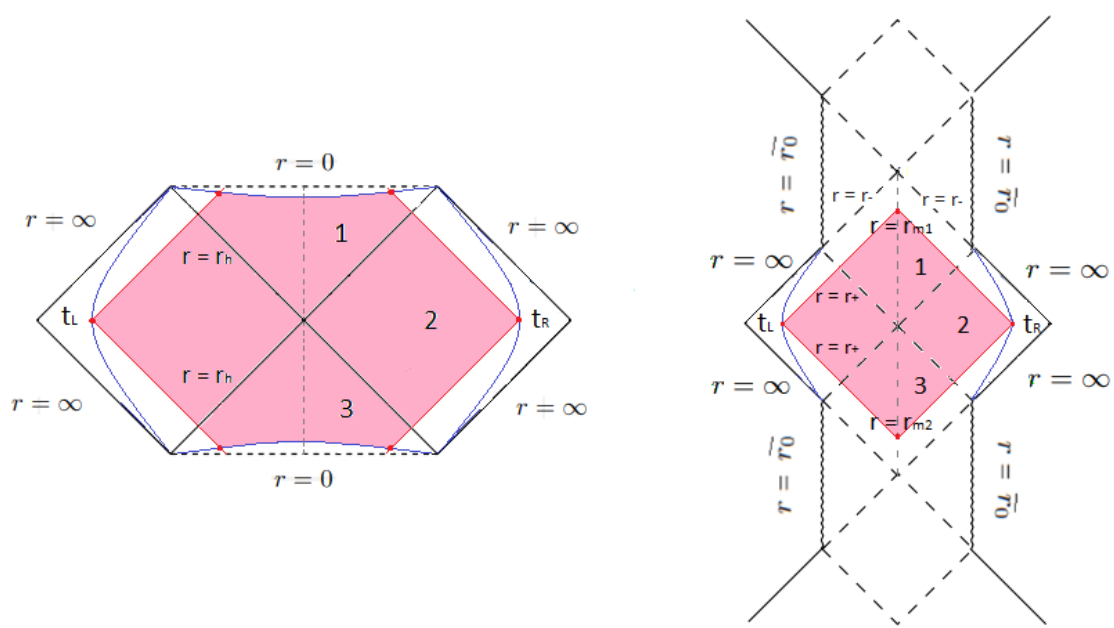

Figure 1. Penrose diagram and WDW patch at $t_{b}=0$ for the non-rotating (left) and rotating (right) black holes.

We are interested in the divergent parts of complexity, so we introduce an UV cutoff at $r=\Lambda$. We will focus on the thermofield double state at time zero, i.e.

$$
t_{L}=t_{R}=t_{b}=0
$$

which by symmetry corresponds to the minimum of the action. The time dependence of the finite part of complexity was previously studied in [62].

The Penrose diagram and the corresponding WDW patch in the non-rotating case are depicted on the left side of figure 1. The configuration of the WDW patch in the rotating case is depicted on the right side of figure 1 . We call $r_{m 1}, r_{m 2}$ the null joints referring respectively to the top and bottom vertices of the spacetime region of interest. The definition of the null joints in terms of the tortoise coordinates are

$$
\frac{t_{b}}{2}+r_{\Lambda}^{*}-r^{*}\left(r_{m 1}\right)=0, \quad \frac{t_{b}}{2}-r_{\Lambda}^{*}+r^{*}\left(r_{m 2}\right)=0,
$$

where $r_{\Lambda}^{*}=r^{*}(\Lambda)$.

Bulk contributions: we follow the calculation in [62]. The integrand of the bulk action is constant, and so this contribution is proportional to the spacetime volume enclosed in the WDW patch. It is convenient to separate this bulk region into three parts, as indicated in figure 1 for the two cases:

$$
\begin{array}{ll}
I_{\mathcal{V}}^{1}=\frac{\mathcal{I}}{8 G} \int_{\bar{r}_{1}}^{r_{+}} d r\left(\frac{t_{b}}{2}+r_{\Lambda}^{*}-r^{*}(r)\right), & I_{\mathcal{V}}^{2}=\frac{\mathcal{I}}{4 G} \int_{r_{+}}^{\Lambda} d r\left(r_{\Lambda}^{*}-r^{*}(r)\right), \\
I_{\mathcal{V}}^{3}=\frac{\mathcal{I}}{8 G} \int_{\bar{r}_{2}}^{r_{+}} d r\left(-\frac{t_{b}}{2}+r_{\Lambda}^{*}-r^{*}(r)\right), &
\end{array}
$$

where $\mathcal{I}$ is defined in eq. (2.13) and the angular part gives a factor of $2 \pi$. The integration range in the non-rotating case is done up to a cutoff $\epsilon_{0} \approx 0$ :

$$
\bar{r}_{1}=\bar{r}_{2}=\varepsilon_{0},
$$


and in the rotating case is:

$$
\bar{r}_{1}=r_{m 1}, \quad \bar{r}_{2}=r_{m 2} .
$$

Boundary terms: in the non-rotating case, we have to include the Gibbons-HawkingYork (GHY) term for the two spacelike boundaries:

$$
I_{\mathcal{B}}=-\frac{1}{8 \pi G} \int_{\mathcal{B}} d^{2} x \sqrt{|h|} K,
$$

where $\mathcal{B}$ is the boundary, $h$ the induced metric determinant, $K$ the trace of the extrinsic curvature. Both in the rotating and non-rotating cases, we have in principle contributions from null boundaries that we set to zero by using an affine parameterization (see eq. (2.17)) for the boundary geodesics [20].

Joint terms: given a joint $\Sigma$ where various boundaries meet, the contribution to the action is given by

$$
I_{\mathcal{J}}=\frac{1}{8 \pi G} \int_{\Sigma} d \theta \sqrt{\sigma} \mathfrak{a},
$$

where $\sigma$ is the determinant of the induced metric on the joint (in this case, it is 1dimensional):

$$
\sqrt{\sigma}=l R(r)=l \sqrt{\frac{r}{4} \Psi(r)} .
$$

The integrand $\mathfrak{a}$ depends on the dot product of normal one-forms $\mathbf{k}_{L}, \mathbf{k}_{R}$ defined on the boundaries meeting at the joint.

All the joints appearing in figure 1 arise from intersections of null lines apart from the ones located at the past and future singularities. However, the latter vanish when we send the IR cutoff to 0 . We will thus focus only on joints where two null lines meet, in which case the integrand is given by

$$
\mathfrak{a}=\eta \log \left|\frac{1}{2} \mathbf{k}_{L} \cdot \mathbf{k}_{R}\right|
$$

where $\eta= \pm 1$ depending on the position of the joint with respect to the future direction of time and the location with respect to the interior of the WDW patch [20]. In our case, the joints in the interior of the black and white holes in figure 1 have $\eta=1$, while the ones nearby the UV cutoff have $\eta=-1$. The null directions are parameterized according to eq. (2.16), which gives

$$
\mathfrak{a}=\eta \log \left|\frac{1}{2} u^{\alpha} v_{\alpha}\right|=\eta \log \left|\frac{A^{2}}{l^{2}} \frac{2 R(r)}{f(r)}\right|,
$$

where $A$ is an overall arbitrary constant which parameterizes the ambiguity in defining the null normals on the boundaries. From eq. (3.9), we find a general expression for all the null joints in the WDW patch

$$
I_{\mathcal{J}}=-\sum_{k} \eta_{k} \frac{l}{4 G} \sqrt{\frac{r_{k}}{4} \Psi\left(r_{k}\right)} \log \left|\frac{l^{2}}{A^{2}} \frac{f\left(r_{k}\right)}{2 R\left(r_{k}\right)}\right|,
$$

where the sum is over the $k$ joints, whose radial coordinate is $r_{k}$. 
Counterterm for the null boundaries: the following counterterm [20] must be added to the boundary term of null boundaries, in order to make the action invariant under reparameterization:

$$
I_{\text {ct }}=\frac{1}{8 \pi G} \int d \theta d \lambda \sqrt{\sigma} \Theta \log |\tilde{L} \Theta|,
$$

where $\lambda$ is the affine parameter of the null geodesics which delimit the boundary, and

$$
\Theta=\frac{1}{\sqrt{\sigma}} \frac{\partial \sqrt{\sigma}}{\partial \lambda}
$$

is the expansion of the congruence of null geodesics on the hypersurface. The parameter $\tilde{L}$ appearing in eq. (3.14) is an arbitrary length scale which is needed for dimensional reasons, whose physical meaning is so far obscure.

We will need to evaluate the counterterm along the null lines described by the null coordinates $(u, v)$. It is convenient to re-express the integral (3.14) in terms of $r$, using

$$
\frac{\partial r}{\partial \lambda}=A v^{r}=\frac{2 A}{l^{2}} R(r) .
$$

Thus, eq. (3.14) becomes

$$
I_{\mathrm{ct}}=\frac{1}{4 G} \int_{r_{\mathrm{inf}}}^{r_{\mathrm{sup}}} d r \frac{\partial \sqrt{\sigma}}{\partial r} \log \left|\frac{\tilde{L}}{\sqrt{\sigma}} \frac{\partial \sqrt{\sigma}}{\partial r} \frac{2 A}{l^{2}} R(r)\right|,
$$

where we integrate between the endpoints along the radial direction of the null line.

\subsection{Total action}

In this section we compute the divergences of the total action of the WDW patch at $t_{b}=0$ in the rotating case. The calculation for the non-rotating case involves slightly different details which are sketched in appendix A.1; as expected, the result reproduces the $r_{-} \rightarrow 0$ limit of the rotating case. The Penrose diagram in the rotating case is depicted in figure 1 , where the top and bottom joint vertices are described by the expressions (3.3). At $t_{b}=0$, we get

$$
r_{\Lambda}^{*}=r^{*}\left(r_{m 1}\right)=r^{*}\left(r_{m 2}\right) \equiv r^{*}\left(r_{m}\right),
$$

and the configuration is symmetric, so the future and past interior actions are the same.

Eqs. (3.18) cannot be solved exactly. Both at $r=\Lambda \rightarrow \infty$ and at $r \rightarrow r_{-}$the function $r^{*}$ diverges to $+\infty$, so we study the behaviour around these points:

- Nearby $r \approx r_{-}$, we find

$$
r^{*}\left(r_{m}\right)=-\frac{\sqrt{3\left(\nu^{2}-1\right)}}{\nu^{2}+3} \tilde{A} \log \left|r_{m}-r_{-}\right|+\tilde{B}+\mathcal{O}\left(r_{m}-r_{-}\right),
$$

where $\tilde{B}$ is a constant and

$$
\tilde{A}=\frac{\sqrt{r_{-} \Psi\left(r_{-}\right)}}{\left(r_{+}-r_{-}\right) \sqrt{3\left(\nu^{2}-1\right)}}>0 .
$$


- Around $r=\Lambda$,

$$
r_{\Lambda}^{*}=\frac{\sqrt{3\left(\nu^{2}-1\right)}}{\nu^{2}+3} \log \Lambda+\tilde{C}+\mathcal{O}\left(\Lambda^{-1}\right),
$$

where $\tilde{C}$ is the finite piece of order $\Lambda^{0}$.

Consequently, eq. (3.18) gives:

$$
r_{m}-r_{-} \approx \Lambda^{-1 / \tilde{A}} \exp \left[\frac{(\tilde{B}-\tilde{C})\left(\nu^{2}+3\right)}{\tilde{A} \sqrt{3\left(\nu^{2}-1\right)}}\right] .
$$

Interior bulk term: the interior bulk term can be obtained from eqs. (3.4), (3.5) and has the following logarithmically divergent piece:

$$
I_{\mathcal{V}}^{\mathrm{int}}=2\left(I_{\mathcal{V}}^{1}+I_{\mathcal{V}}^{3}\right)=-\frac{l}{4 G}\left(\nu^{2}+3\right)\left[\left(r_{+}-r_{m}\right) r_{\Lambda}^{*}-\int_{r_{m}}^{r_{+}} d r r^{*}(r)\right]
$$

The last integral in eq. (3.23) is finite, because the function $r^{*}(r)$ has integrable singularities in $r \approx r_{-}, r_{+}$. The divergent part of the internal bulk action is:

$$
I_{\mathcal{V}}^{\text {int }}=-\frac{l}{4 G} \sqrt{3\left(\nu^{2}-1\right)}\left(r_{+}-r_{-}\right) \log \Lambda+\mathcal{O}\left(\Lambda^{0}\right) .
$$

External bulk term: we then consider the external part

$$
I_{\mathcal{V}}^{\text {ext }}=2 I_{\mathcal{V}}^{2}=-\frac{l}{4 G}\left(\nu^{2}+3\right) \int_{r_{+}}^{\Lambda} d r\left(r_{\Lambda}^{*}-r^{*}(r)\right)
$$

The behaviour of $r^{*}(r)$ at large $r$ is:

$$
r^{*}(r)=\alpha \log (4 r)+\beta+\frac{\gamma}{r}+\mathcal{O}\left(r^{-2}\right)
$$

where

$$
\begin{aligned}
& \beta=-2 \frac{\sqrt{r_{+} \Psi\left(r_{+}\right)} \log \left(\sqrt{r_{+}}+\sqrt{r_{+}-\rho_{0}}\right)-\sqrt{r_{-} \Psi\left(r_{-}\right)} \log \left(\sqrt{r_{-}}+\sqrt{r_{-}-\rho_{0}}\right)}{\left(\nu^{2}+3\right)\left(r_{+}-r_{-}\right)}, \\
& \alpha=\frac{\sqrt{3\left(\nu^{2}-1\right)}}{\nu^{2}+3}, \quad \gamma=\frac{\sqrt{3\left(\nu^{2}-1\right)}}{2\left(\nu^{2}+3\right)}\left(\rho_{0}-2 r_{+}-2 r_{-}\right) .
\end{aligned}
$$

The divergences of (3.25) then are

$$
\begin{aligned}
I_{\mathcal{V}}^{\mathrm{ext}} & =\frac{l}{4 G}\left(\nu^{2}+3\right)\left[-\alpha \Lambda+\left(\alpha r_{+}+\gamma\right) \log \Lambda\right]+\mathcal{O}\left(\Lambda^{0}\right) \\
& =-\Lambda \frac{l}{4 G} \sqrt{3\left(\nu^{2}-1\right)}+\frac{l}{8 G} \sqrt{3\left(\nu^{2}-1\right)}\left(\rho_{0}-2 r_{-}\right)(\log \Lambda)+\mathcal{O}\left(\Lambda^{0}\right)
\end{aligned}
$$


Joint terms: the action evaluated on the WDW patch has four joint contributions: two on the cutoff surface $r=\Lambda$ and two in the region inside the black and white hole. They can all be directly evaluated from eq. (3.13). The joint inside the black hole, located at $r=r_{m}$, gives the following contribution:

$$
\begin{aligned}
I_{\mathcal{J}}^{r_{m}} & =-\frac{l}{8 G} \sqrt{r_{m} \Psi\left(r_{m}\right)} \log \left|\frac{l^{2}}{A^{2}} \frac{\left(\nu^{2}+3\right)\left(r_{m}-r_{-}\right)\left(r_{m}-r_{+}\right)}{r_{m} \Psi\left(r_{m}\right)}\right| \\
& =\frac{l}{8 G} \sqrt{3\left(\nu^{2}-1\right)}\left(r_{+}-r_{-}\right) \log \Lambda+\mathcal{O}\left(\Lambda^{0}\right) .
\end{aligned}
$$

The joint nearby the cutoff surface gives:

$$
\begin{aligned}
I_{\mathcal{J}}^{\Lambda} & =\frac{l}{8 G} \sqrt{\Lambda \Psi(\Lambda)} \log \left|\frac{l^{2}}{A^{2}} \frac{\left(\nu^{2}+3\right)\left(\Lambda-r_{-}\right)\left(\Lambda-r_{+}\right)}{\Lambda \Psi(\Lambda)}\right| \\
& =\Lambda \frac{l}{8 G} \sqrt{3\left(\nu^{2}-1\right)} \log \left|\frac{l^{2}}{A^{2}} \frac{\nu^{2}+3}{3\left(\nu^{2}-1\right)}\right|+\mathcal{O}\left(\Lambda^{0}\right) .
\end{aligned}
$$

Summing the contributions of the four joints, we find:

$$
I_{\mathcal{J}}^{\text {tot }}=\Lambda \frac{l}{4 G} \sqrt{3\left(\nu^{2}-1\right)} \log \left|\frac{l^{2}}{A^{2}} \frac{\nu^{2}+3}{3\left(\nu^{2}-1\right)}\right|+\frac{l}{4 G} \sqrt{3\left(\nu^{2}-1\right)}\left(r_{+}-r_{-}\right) \log \Lambda+\mathcal{O}\left(\Lambda^{0}\right) .
$$

Counterterm: the WDW patch is bounded by four codimension-1 null surfaces; they are all the same by symmetry, and so from (3.17) and (3.10) we find:

$$
I_{\mathrm{ct}}=\frac{l}{4 G} \int_{r_{m}}^{\Lambda} d r \frac{\partial_{r}(r \Psi(r))}{\sqrt{r \Psi(r)}} \log \left|\frac{2 A \tilde{L}}{l^{2}} \frac{\partial_{r}(r \Psi(r))}{4 \sqrt{r \Psi(r)}}\right| .
$$

Since $\Psi(r)$ is linear in $r$, the only divergence comes from $r=\Lambda$ :

$$
I_{\mathrm{ct}}=\Lambda \frac{l}{4 G} \sqrt{3\left(\nu^{2}-1\right)} \log \left|\frac{\tilde{L}^{2} A^{2}}{l^{4}} 3\left(\nu^{2}-1\right)\right|+\mathcal{O}\left(\Lambda^{0}\right) .
$$

Total action: summing all the contributions, the divergences of the total action are:

$$
I^{\mathrm{tot}}=\frac{l}{4 G} \sqrt{3\left(\nu^{2}-1\right)} \Lambda\left(\log \left(\frac{\tilde{L}^{2}}{l^{2}}\left(\nu^{2}+3\right)\right)-1\right)+(\log \Lambda) \frac{l}{4 G} \sqrt{3\left(\nu^{2}-1\right)}\left(\frac{\rho_{0}}{2}-r_{-}\right),
$$

As in the AdS case, the divergent contribution in the counterterm cancels the dependence on the ambiguous normalization constant $A$ appearing in the divergent contribution of the joints.

\subsection{Action of internal region and subregion complexity}

In this section we compute the divergences of the action evaluated on the intersection between the WDW patch and the interior of the black and white holes. The external part, which is conjectured to be proportional to the subregion complexity of the thermofield double state, is then found by subtraction from the total WDW action. Again we consider the rotating black hole; the non-rotating case is studied in appendix A.2. The bulk part of the internal action was already computed in (3.24). 


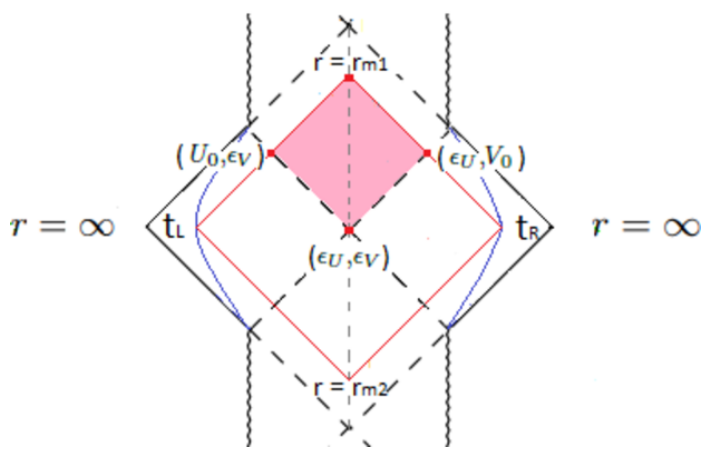

Figure 2. Joint terms needed for the action of the black hole interior.

Joint terms: in the interior of the black hole, there are four contributions of the form (3.13), which are all in principle divergent because $f\left(r_{+}\right)=f\left(r_{-}\right)=0$. Symmetrically, there are other four joints inside the white hole. As in the AdS case [46], due to the signs $\eta= \pm 1$ of each joint, these divergences will partially cancel each other.

It is useful to introduce the Kruskal coordinates $(U, V)$ defined for $r>r_{-}$as in [71]

$$
\begin{aligned}
& U=\operatorname{sgn}\left(r-r_{+}\right) e^{b_{*}\left(r^{*}(r)-t\right)}=\operatorname{sgn}\left(r-r_{+}\right) e^{-b_{*} u}, \\
& V=e^{b_{*}\left(r^{*}(r)+t\right)}=e^{b_{*} v},
\end{aligned}
$$

where

$$
b_{*}=\frac{f^{\prime}\left(r_{+}\right)}{2}=\frac{\left(\nu^{2}+3\right)\left(r_{+}-r_{-}\right)}{2 \sqrt{r_{+} \Psi\left(r_{+}\right)}} .
$$

These coordinates satisfy the relation

$$
\log |U V|=2 b_{*} r^{*}(r)=f^{\prime}\left(r_{+}\right) r^{*}(r)
$$

which is useful to simplify expressions involving the joints. Note that, since $r_{*} \rightarrow-\infty$ when $r \rightarrow r_{+}$, the external horizon corresponds to $U=0$ (black hole horizon for the right boundary) and $V=0$ (white hole horizon for the right boundary).

Let us consider a contribution coming from sums of joints nearby the horizon. We follow the prescription given in [46], introducing the regulators $\varepsilon_{U}, \varepsilon_{V}$ to move the joints off the horizon by an infinitesimal quantity. For instance, if we evaluate the sum of the contributions of two terms with the same $V=\varepsilon_{V}$, from eq. (3.13), we find a term proportional to

$$
\begin{aligned}
\log \left|\frac{l^{2}}{A^{2}} \frac{f\left(r_{U_{1}, \varepsilon_{V}}\right)}{2 R\left(r_{+}\right)}\right|-\log \left|\frac{l^{2}}{A^{2}} \frac{f\left(r_{U_{2}, \varepsilon_{V}}\right)}{2 R\left(r_{+}\right)}\right| & =\int_{r_{U_{2}, \varepsilon_{V}}}^{r_{U_{1}, \varepsilon_{V}}} \frac{d r}{f(r)} f^{\prime}(r) \approx f^{\prime}\left(r_{+}\right) \int_{r_{U_{2}, \varepsilon_{V}}}^{r_{U_{1}, \varepsilon_{V}}} \frac{d r}{f(r)} \\
& =f^{\prime}\left(r_{+}\right)\left[r^{*}\left(r_{U_{1}, \varepsilon_{V}}\right)-r^{*}\left(r_{U_{2}, \varepsilon_{V}}\right)\right] \\
& =\log \left|U_{1} \varepsilon_{V}\right|-\log \left|U_{2} \varepsilon_{V}\right|=\log \left|\frac{U_{1}}{U_{2}}\right|
\end{aligned}
$$

where in the last steps we simplified the result by means of eq. (3.37). 
This expression tells us that in the limit $\varepsilon_{V} \rightarrow 0$, the difference of joints at the horizon is regular and the divergences coming from each term separately cancel. We could perform the same trick exchanging the $U \leftrightarrow V$ coordinates, since the previous manipulations are symmetric under this transformation. Combining these two results, one can conclude that

$$
\log \left|\frac{l^{2}}{A^{2}} \frac{f\left(r_{U, V)}\right.}{2 R\left(r_{+}\right)}\right|=\log |U V|+F\left(r_{+}\right)
$$

where the function $F(r)$ is regular at the horizon and is given by

$$
F(r)=\log \left|\frac{l^{2}}{A^{2}} \frac{f(r)}{2 R(r)}\right|-f^{\prime}\left(r_{+}\right) r^{*}(r) .
$$

There are four joint contributions inside the black hole and four inside the white hole; by symmetry they are the same and the total contribution is twice the ones of the black hole:

$$
\begin{aligned}
I_{\mathcal{J}}^{\text {int }}= & -2 \times \frac{l}{4 G} \sqrt{\frac{r_{+}}{4} \Psi\left(r_{+}\right)}\left[\log \left|\frac{l^{2}}{A^{2}} \frac{f\left(r_{\epsilon_{U}, \epsilon_{V}}\right)}{2 R\left(r_{+}\right)}\right|-\log \left|\frac{l^{2}}{A^{2}} \frac{f\left(r_{U_{0}, \epsilon_{V}}\right)}{2 R\left(r_{+}\right)}\right|-\log \left|\frac{l^{2}}{A^{2}} \frac{f\left(r_{\epsilon_{U}, V_{0}}\right)}{2 R\left(r_{+}\right)}\right|\right] \\
& -2 \times \frac{l}{4 G} \sqrt{\frac{r_{m}}{4} \Psi\left(r_{m}\right)} \log \left|\frac{l^{2}}{A^{2}} \frac{f\left(r_{m}\right)}{2 R\left(r_{m}\right)}\right|
\end{aligned}
$$

This expression simplifies to

$$
\begin{aligned}
I_{\mathcal{J}}^{\text {int }} & =\frac{l}{4 G} \sqrt{r_{+} \Psi\left(r_{+}\right)}\left[2 b_{*} r_{\Lambda}^{*}+F\left(r_{+}\right)\right]-\frac{l}{4 G} \sqrt{r_{m} \Psi\left(r_{m}\right)} \log \left|\frac{l^{2}}{A^{2}} \frac{f\left(r_{m}\right)}{2 R\left(r_{m}\right)}\right| \\
& =\frac{l}{2 G} \sqrt{3\left(\nu^{2}-1\right)}\left(r_{+}-r_{-}\right) \log \Lambda+\mathcal{O}\left(\Lambda^{0}\right) .
\end{aligned}
$$

Counterterms: the last contribution comes from the counterterm, and possible dependences from the UV cutoff can arise only from the $r=r_{m}$ endpoint of integration. However, putting the expansion (3.22) inside the counterterm, we find that no divergent pieces appear.

Internal and external action: putting together all the terms contributing to the interior action in the rotating case, we find that the divergent part of the internal action is:

$$
I^{\mathrm{int}}=\frac{l}{4 G} \sqrt{3\left(\nu^{2}-1\right)}\left(r_{+}-r_{-}\right) \log \Lambda+\mathcal{O}\left(\Lambda^{0}\right) .
$$

Subtracting this from eq. (3.34), we find the divergences of the external action, which correspond to the subsystem complexity:

$$
I^{\mathrm{ext}}=\frac{l}{4 G} \sqrt{3\left(\nu^{2}-1\right)} \Lambda\left(\log \left(\frac{\tilde{L}^{2}}{l^{2}}\left(\nu^{2}+3\right)\right)-1\right)+(\log \Lambda) \frac{l}{4 G} \sqrt{3\left(\nu^{2}-1\right)}\left(\frac{\rho_{0}}{2}-r_{+}\right) .
$$




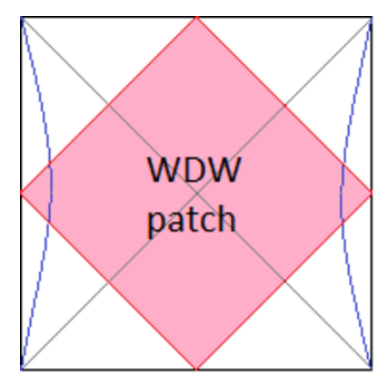

Regularization A

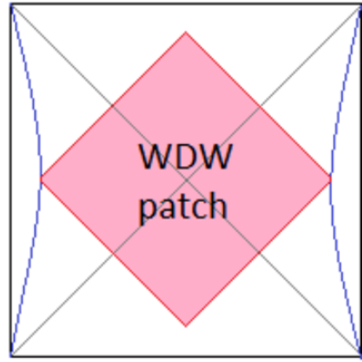

Regularization B

Figure 3. Two different regularizations can be chosen for the action of the WDW patch for black holes in AdS (here for illustrative purpose we show the case of non-rotating black hole in asymptotically AdS spacetime).

\subsection{Comments on regularization}

In AdS one can use two different regularizations [39] for the CA conjecture (see figure 3):

- one can consider the edge of the WDW ending on the asymptotic AdS boundary (regularization $A$ )

- one can consider the edge ending on the regulator surface (regularization $B$ ).

The two regularizations give the same complexity rate at large times. In asymptotically AdS spaces, if one introduces appropriate counterterms in regularization $A$ one can reproduce the same results as in regularization $B[72,73]$.

In WAdS the structure of the Penrose diagram is radically different from AdS, and it resembles instead the one of asymptotically Minkowski space: the right corner of the Penrose diagram corresponds to $r \rightarrow \infty$ and arbitrary $t$ (spacelike infinity). The 45 degrees boundaries correspond to the future null infinity and past null infinity (see figure 4).

In all the previous works on the CA conjectures in WAdS [60, 62, 63], regularization $B$ was implicitly used. This approach gives the expected result for the complexity rate at late time $\dot{\mathcal{C}}_{A} \propto T S$ in the case of Einstein gravity [62]. We used as well this regularization in the previous section to compute subregion action complexity.

It is not straightforward to generalise regularization $A$ to the case of WAdS, because then the corner of the WDW patch would be located at the spacelike infinity point for all values of the time. This would give the unphysical result that complexity is timeindependent.

If in regularization $B$ we sent the UV cutoff to infinity, we would find that the WDW patch includes all the interior of the black hole. This is the same part of the Penrose diagram which gives the linear growth of complexity at large time; so sending the cutoff to infinity is equivalent to sending the time to infinity with finite cutoff, and so gives a diverging internal action. This explains why the action of the internal part of the WDW is UV divergent in WAdS, while it is finite in AdS. 


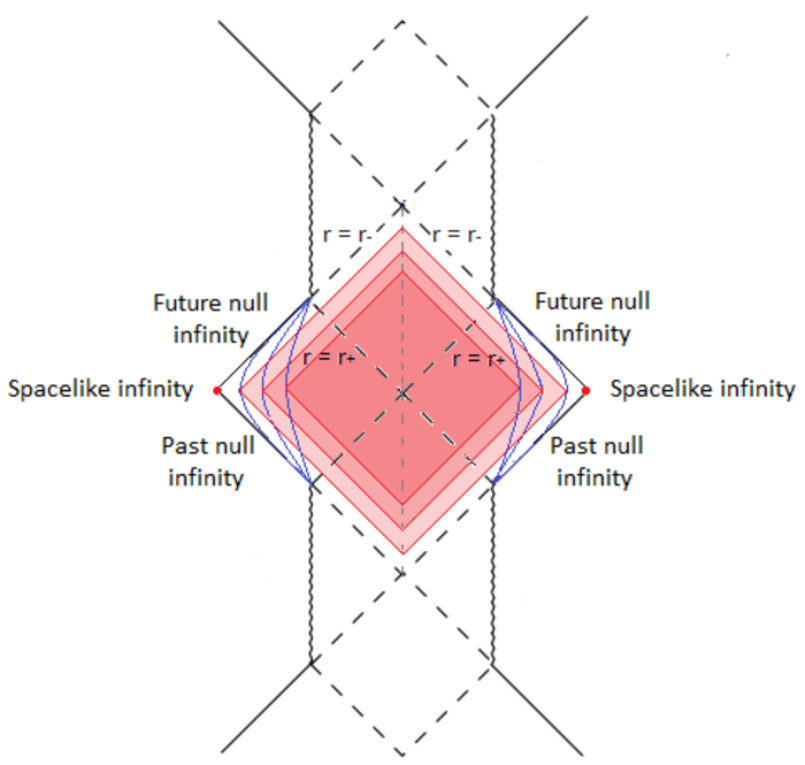

Figure 4. In WAdS the causal structure resembles the one of asymptotically Minkowski spacetime. Regularization $A$ would give a WDW patch with a corner which is located at the spacelike infinity, and so would give a time-independent complexity. Moreover, the WDW patch in regularization $B$ covers the entire black hole interior in the limit of infinite cutoff $\Lambda$.

\subsection{Comments on counterterms}

Following [20], we inserted in the gravitational action a counterterm of kind (3.14) which is needed in order to maintain reparameterization invariance in presence of null boundaries. This term is not necessarily unique.

Let us borrow some notation from [20]. Let us consider a null hypersurface defined by the function $\Phi\left(x^{\alpha}\right)=0$. The hypersurface can be described by parametric equations $x^{\mu}\left(\lambda, \theta^{A}\right)$, where $\lambda$ is the affine null parameter and $\theta^{A}$ is constant on each null generator on the surface. The vectors:

$$
k^{\mu}=\frac{\partial x^{\mu}}{\partial \lambda}, \quad e_{A}^{\mu}=\frac{\partial x^{\mu}}{\partial \theta^{A}},
$$

are tangent to the surface; $k^{\alpha}$ is the null normal to the surface. Let us denote by

$$
\sigma_{A B}=g_{\alpha \beta} e_{A}^{\alpha} e_{B}^{\beta}
$$

the induced metric on the transverse directions $\theta_{A}$. Also, one can introduce the following tensor

$$
B_{A B}=e_{A}^{\alpha} e_{B}^{\beta} D_{\alpha} k_{\beta},
$$

which describes the behaviour of the congruence of null generators.

In principle, as discussed in the appendix B of [20], in presence of null boundaries we can also allow for Lagrangians depending on combinations of the Riemann tensor $\hat{R}_{A B C D}$ computed from the transverse induced metric $\sigma_{A B}$. Moreover, contributions containing the tensor $B_{A B}$ are also allowed. A priori we could have a counterterm of the type

$$
\mathcal{L}_{\mathrm{ct}}\left(\hat{R}, \hat{R}_{A B}, \hat{R}_{A B C D}, B_{A B}, \Theta\right),
$$


where we should require that the total action is reparametrization-invariant. Dramatic restrictions arise from the fact that we are working in 3 dimensions, which means that the null surfaces are 2-dimensional and that the induced metric $\sigma_{A B}$ is 1-dimensional. This implies that

$$
\hat{R}_{A B C D}=0, \quad \hat{R}_{A B}=0, \quad \hat{R}=0, \quad B_{A B}=\frac{1}{2} \Theta \sigma_{A B},
$$

and then there is no space for curvature terms other than the geodesic expansion parameter $\Theta$, which we already considered for the counterterm (3.14).

\section{Volume}

\subsection{CV conjecture}

In this section we compute the divergences of the volume complexity at $t_{b}=0$ for the generic rotating black hole (the non-rotating case is studied in appendix A.3, where also the finite part is evaluated). The time dependence of the finite part of the volume was previously studied in [61].

The extremal volume at $t_{b}=0$ is a constant $t=0$ bulk slice, connecting the two $t_{L}=0$ and $t_{R}=0$ regions on the left and right boundaries. The RT surface is a line at a constant value of the radial coordinate $r=r_{+}$. We denote by $V(L)$ the volume of the codimension-1 extremal surface anchored at the entire left boundary of the spacetime, and by $V(R)$ the corresponding volume for the right boundary. The symmetry of the problem implies that the subregion complexity on the two boundaries separately is the same, and then

$$
V^{\text {out }}=V(L)+V(R)=2 V(L) .
$$

The volume can be computed directly from the determinant of the induced metric on the $t=0$ slice:

$$
\begin{aligned}
V(L) & =2 \pi l^{2} \int_{r_{+}}^{\Lambda} d r G(r), \\
G(r) & =\sqrt{\frac{r\left(3\left(\nu^{2}-1\right) r+\left(\nu^{2}+3\right)\left(r_{+}+r_{-}\right)-4 \nu \sqrt{r_{+} r_{-}\left(\nu^{2}+3\right)}\right)}{4\left(\nu^{2}+3\right)\left(r-r_{-}\right)\left(r-r_{+}\right)}} .
\end{aligned}
$$

Let us study the possible divergences of this integral.

Nearby the outer horizon $r \rightarrow r_{+}, G(r)$ can be approximated by the following expression:

$G(r)=\frac{g}{\sqrt{r-r_{+}}}+\mathcal{O}\left(\sqrt{r-r_{+}}\right), \quad g=\sqrt{\frac{r_{+}\left(4 \nu^{2} r_{+}+\left(\nu^{2}+3\right) r_{-}-4 \nu \sqrt{r_{+} r_{-}\left(\nu^{2}+3\right)}\right)}{4\left(\nu^{2}+3\right)\left(r_{+}-r_{-}\right)}}$.

The contribution to the volume coming from the region nearby the outer horizon is:

$$
2 \pi l^{2} \int_{r_{+}}^{r_{+}+\varepsilon} d r G(r) \approx 2 \pi l^{2} \int_{r_{+}}^{r_{+}+\varepsilon} d r \frac{g}{\sqrt{r-r_{+}}} \approx 4 \pi l^{2} g \sqrt{\epsilon} .
$$

So there is no divergence nearby the horizon. 
At $r \rightarrow \infty, G(r)$ can be expanded as follows:

$$
G(r)=\sqrt{\frac{3\left(\nu^{2}-1\right)}{4\left(\nu^{2}+3\right)}}+\frac{\nu\left(\nu\left(r_{+}+r_{-}\right)-\sqrt{r_{+} r_{-}\left(\nu^{2}+3\right)}\right)}{\sqrt{3\left(\nu^{2}-1\right)\left(\nu^{2}+3\right)}} \frac{1}{r}+\mathcal{O}\left(\frac{1}{r^{2}}\right) .
$$

Upon integration, the first two terms give rise to a linear and a logarithmic divergences. The divergence of the volume then is

$$
V(L)=\pi l^{2} \sqrt{\frac{3\left(\nu^{2}-1\right)}{\nu^{2}+3}} \Lambda+\frac{32 \pi G l^{2} \nu^{2}}{\left(\nu^{2}+3\right)^{3 / 2} \sqrt{3\left(\nu^{2}-1\right)}} M \log \Lambda+\mathcal{O}\left(\Lambda^{0}\right) .
$$

The logarithmically divergent term is proportional to the mass $M$.

\subsection{Spacetime volume (CV 2.0)}

It was proposed in [21] that complexity is dual to the spacetime volume of the WDW patch; in our case this is very similar to the action conjecture because the bulk term in the action is the integral of a constant. We can borrow the calculations of the divergences from eqs. (3.24) and (3.28):

$$
\begin{aligned}
& V_{\text {bulk }}^{\text {int }}=4 \pi l^{3} \frac{\sqrt{3\left(\nu^{2}-1\right)}}{\nu^{2}+3}\left(r_{+}-r_{-}\right) \log \Lambda+\mathcal{O}\left(\Lambda^{0}\right), \\
& V_{\text {bulk }}^{\text {ext }}=4 \pi l^{3} \frac{\sqrt{3\left(\nu^{2}-1\right)}}{\nu^{2}+3}\left(\Lambda-\left(\frac{\rho_{0}}{2}-r_{-}\right) \log \Lambda\right)+\mathcal{O}\left(\Lambda^{0}\right) .
\end{aligned}
$$

\section{Conclusions}

Sub/superadditivity. In AdS black holes the internal action $I^{\text {int }}$ at $t_{b}=0$ is finite $[46,47]$ and has a sign which depends on the choice of the counterterm parameter $\tilde{L}$. In turn, depending on the sign of $I^{\text {int }}$, the action subregion complexity can be sub/superadditive. Instead, in $\mathrm{WAdS}_{3}$ the interior action $I^{\text {int }}$ is always positive and independent of the counterterm length scale; as a consequence, $\mathcal{C}_{A}$ subregion complexity of the left and right side of the thermofield double is superadditive. Moreover, $I^{\text {int }}$ is proportional to the product of temperature and entropy of the black hole:

$$
I^{\text {int }}=\frac{4 \sqrt{3\left(\nu^{2}-1\right)}}{\nu^{2}+3} l T S \log \Lambda+\mathcal{O}\left(\Lambda^{0}\right) .
$$

By construction, $\mathcal{C}_{V}$ and $\mathcal{C}_{V} 2.0$ are superadditive, with the difference that $\mathcal{C}_{V}$ saturates superadditivity (1.5) for the left and right side of thermofield double at $t_{b}=0$, while $\mathcal{C}_{V}$ 2.0 does not, see eq. (4.7).

Structure of divergences. For the BTZ black hole, the only divergence in the holographic subregion complexity is linear in the cutoff $\Lambda$. In $\mathrm{WAdS}_{3}$, we found that the three versions of holographic subregion complexity have all a linear and a logarithmic divergence in $\Lambda$. The coefficient of the linear divergence, as in the BTZ case, can be positive or negative depending on the counterterm parameter $\tilde{L}$. The coefficient of the log divergence 
is independent from $\tilde{L}$; it is instead a function of the black holes parameters $r_{+}, r_{-}$, or equivalently of $T, J$. In each of the three versions, the log divergence of the subregion complexity is proportional to a different quantity:

- in the CA conjecture, eq. (3.44) gives a result proportional to $K_{+}=\frac{\rho_{0}}{2}-r_{+}$, with a positive coefficient;

- in the CV conjecture, eq. (4.6) gives a term proportional to the mass $M$, with a positive coefficent;

- in CV 2.0, eq. (4.7) gives a log divergence proportional to $K_{-}=\frac{\rho_{0}}{2}-r_{-}$, with a negative coefficient.

Temperature behaviour. For neutral black holes in AdS, subregion $\mathcal{C}_{A}$ has different properties depending on the regularization parameter $\tilde{L}$. For $\tilde{L} \ll l, \mathcal{C}_{A}$ increases with temperature, whereas, for $\tilde{L} \gg l, \mathcal{C}_{A}$ decreases with temperature. Instead, for neutral black holes in AdS, subregion $\mathcal{C}_{V}$ is an increasing powerlike function of temperature [25] (for $\mathrm{AdS}_{3}$, actually, it is independent of temperature).

In $\mathrm{WAdS}_{3}$, the leading dependence on temperature of the subsystem complexity is in the $\log \Lambda$ terms. To this purpose we introduce

$$
C_{J}=\left.\frac{\partial M}{\partial T}\right|_{J}, \quad C_{+}=\left.\frac{\partial K_{+}}{\partial T}\right|_{J}, \quad C_{-}=\left.\frac{\partial K_{-}}{\partial T}\right|_{J},
$$

which are explicitly computed in appendix B. $C_{J}$ is the specific heat at constant $J$. We note that the scale $r_{+}$factorises from the quantities (5.2), so we introduce

$$
\epsilon=r_{-} / r_{+}, \quad 0 \leq \epsilon<1
$$

and we study the sign of (5.2) as a function of $(\epsilon, \nu)$. Let us define (see figure 5)

$$
\begin{array}{ll}
\text { Region A : } & 0<\epsilon<\frac{\nu^{2}+3}{4 \nu^{2}} \equiv \epsilon_{c}(\nu) \\
\text { Region B : } & \epsilon_{c}(\nu)<\epsilon<1 .
\end{array}
$$

The angular momentum $J$ is negative in region $\mathrm{A}$ and positive in region $\mathrm{B}$, while it vanishes along the two curves $\epsilon=0$ and $\epsilon=\epsilon_{c}(\nu)$. It is interesting that the quantities $\left(C_{J}, C_{+}\right)$change sign in regions $A, B$ :

- $C_{J}$ is positive in region $B$ and negative in region $A$;

- $C_{+}$is negative in region $B$ and positive in region $A$.

As a consequence, in the region where $C_{J}>0$, subregion $\mathcal{C}_{V}$ increases with temperature (at constant $J$ ), while $\mathcal{C}_{A}$ decreases. In the thermodynamically unstable region where $C_{J}<0$, subregion $\mathcal{C}_{V}$ decreases with temperature while $\mathcal{C}_{A}$ increases.

The behaviour of spacetime volume $\mathcal{C}_{V}^{2.0}$ with temperature is shown in figure 6 , where the region $A$ is still the one defined in eq. (5.5) and region $B$ splits into regions $B_{1}$ and $B_{2}$. 


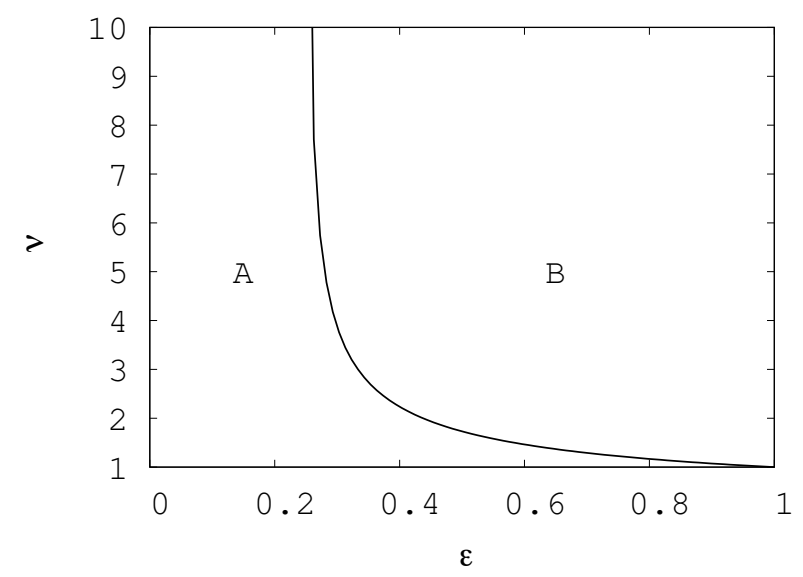

Figure 5. Regions A and B in $(\epsilon, \nu)$ plane. The angular momentum $J$ and the specific heat $C_{J}$ are negative in region $A$ and positive in region $B . \mathcal{C}_{V}$ decreases with temperature in region $A$ and increases in region $B . \mathcal{C}_{A}$ increases with temperature in region $A$ and decreases in region $B$.

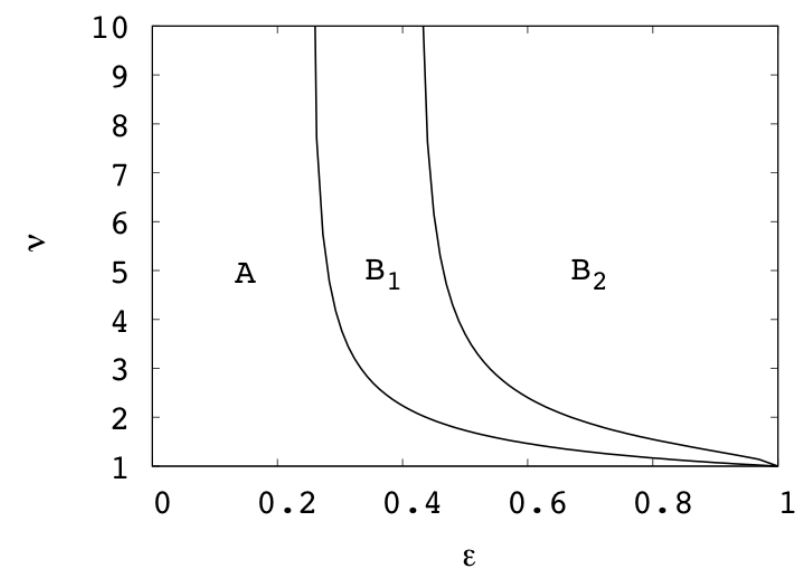

Figure 6. Regions of $(\epsilon, \nu)$ with different temperature behaviour of subregion $\mathcal{C}_{V}^{2.0}$ : it decreases with temperature at constant $J$ in regions $A$ and $B_{2}$, while it increases in region $B_{1}$.

Subregion spacetime volume decreases with temperature at constant $J$ in regions $A$ and $B_{2}$, while it increases in region $B_{1}$. Note that the three quantities $\left(C_{J}, C_{+}, C_{-}\right)$all diverge along the curves $\epsilon=0$ and $\epsilon=\epsilon_{c}(\nu)$.

In this work we find that in $\mathrm{WAdS}_{3}$ the properties of $\mathcal{C}_{A}$ are somehow more robust compared to the AdS case, because they do not depend on the value of $\tilde{L}: \mathcal{C}_{A}$ is universally superadditive for the $L, R$ states of the TD state and the behaviour of subregion complexity with temperature is correlated with the specific heat.

On general grounds, $\mathcal{C}_{B}$ is expected to decrease with $T$ [46]. The sub/superadditivity properties of subregion $\mathcal{C}_{P}$ and $\mathcal{C}_{B}$ are not firmly established and further studies are desirable. According to [46], $\mathcal{C}_{P}$ should be subadditive for the $L$ and $R$ factors of $T D$, while $\mathcal{C}_{B}$ should be superadditive (see however [48] for an independent discussion which suggests that purification complexity might be neither sub nor superadditive). Our findings for 
$\mathcal{C}_{A}$ seem to match these expectations for $\mathcal{C}_{B}$ in the thermodynamically stable region with $C_{J}>0$. On the contrary, $\mathcal{C}_{V}$ seems to match with $\mathcal{C}_{B}$ in the unstable region with $C_{J}<0$.

The physical meaning of the scale $\tilde{L}$ in the CA conjecture is still rather obscure. In AdS many properties of subregion complexity are sensitive to this parameter. It is possible that $\tilde{L}$ is somehow related to the regularization procedure needed to define complexity in quantum field theory; it would be interesting to study this issue. In $\mathrm{WAdS}_{3}$ instead the scale $\tilde{L}$ has less influence on the physical properties of holographic complexity.

The properties of purification and basis complexity deserve further investigation, in order to understand if some universal features can emerge which can be matched with general features of holographic complexity. It would be also interesting to search for other measures of mixed state quantum complexity that may have different properties.

\section{Acknowledgments}

AM acknowledges the financial support from a Fellowship from the ICTP Programme for TRIL, Trieste, Italy and the hospitality of UCSC, Brescia, Italy, while this work was in progress.

\section{A Non-rotating case}

In this appendix we consider in detail the non-rotating case with $r_{+}=r_{h}$ and $r_{-}=0$ and we check that the divergences of complexity reproduce the appropriate limit of the rotating case.

\section{A.1 Total action}

Summing eqs. (3.4), (3.5) the bulk action is

$$
I_{\mathcal{V}}^{\mathrm{tot}}=\frac{\mathcal{I}}{2 G} \int_{0}^{\Lambda} d r\left(r_{\Lambda}^{*}-r^{*}(r)\right)=-\frac{l}{4 G}\left(\nu^{2}+3\right) \Lambda r_{\Lambda}^{*}+\frac{l}{4 G}\left(\nu^{2}+3\right) \int_{0}^{\Lambda} d r r^{*}(r) .
$$

The GHY term (3.8) gives [62]:

$$
I_{\mathcal{B}}=-\frac{\left(\nu^{2}+3\right) l}{4 G}\left(2 \varepsilon_{0}-r_{h}\right)\left(r_{\Lambda}^{*}-r^{*}\left(\varepsilon_{0}\right)\right)=\frac{\left(\nu^{2}+3\right) l}{4 G} r_{h}\left(r_{\Lambda}^{*}-r^{*}(0)\right),
$$

where in the last step we performed the limit $\varepsilon_{0} \rightarrow 0$. The expression is divergent after sending $\Lambda \rightarrow \infty$ due to the behaviour at infinity of the tortoise coordinate.

At $t_{b}=0$, the joints of the WDW patch are located at both the IR and UV cutoffs. The former vanish as already observed, while the latter give the only non-vanishing expression. If we conventionally decide to take the flow of time in the bulk as increasing when going upwards, these joints take a negative sign $\eta=-1$ in eq. (3.13) and we obtain

$$
I_{\mathcal{J}}=2 \times \frac{l}{4 G} \sqrt{\frac{\Lambda}{4} \Psi(\Lambda)} \log \left|\frac{l^{2}}{A^{2}} \frac{f(\Lambda)}{2 R(\Lambda)}\right|=\frac{l}{4 G} \sqrt{\Lambda \Psi(\Lambda)} \log \left|\frac{l^{2}}{A^{2}} \frac{\left(\nu^{2}+3\right)\left(\Lambda-r_{h}\right)}{\Psi(\Lambda)}\right| .
$$


Finally, we have to add the counterterm which renders the action diffeomorphism-invariant:

$$
I_{\mathrm{ct}}=4 \times \frac{l}{4 G} \int_{\varepsilon_{0}}^{\Lambda} d r \frac{6\left(\nu^{2}-1\right) r+\left(\nu^{2}+3\right) r_{h}}{4 \sqrt{r \Psi(r)}} \log \left|\frac{A \tilde{L}}{2 l^{2}} \frac{6\left(\nu^{2}-1\right) r+\left(\nu^{2}+3\right) r_{h}}{\sqrt{r \Psi(r)}}\right| .
$$

The integration can be done analytically and we can also perform the usual limit $\varepsilon_{0} \rightarrow 0$, finding

$$
\begin{aligned}
I_{\mathrm{ct}}=\frac{l}{4 G}\left[\frac{2\left(\nu^{2}+3\right) r_{h}}{\sqrt{3\left(\nu^{2}-1\right)}} \arctan \left(\frac{\sqrt{3\left(\nu^{2}-1\right) \Lambda}}{\sqrt{\left(\nu^{2}+3\right) r_{h}+3\left(\nu^{2}-1\right) \Lambda}}\right)\right. \\
\left.-\sqrt{\Lambda \Psi(\Lambda)} \log \left|\frac{4 l^{4}}{A^{2} \tilde{L}^{2}} \frac{\Lambda \Psi(\Lambda)}{\left(\left(\nu^{2}+3\right) r_{h}+6\left(\nu^{2}-1\right) \Lambda\right)^{2}}\right|\right] .
\end{aligned}
$$

Putting all these results together we obtain the expression for the total action in the WDW patch

$$
\begin{aligned}
I^{\mathrm{tot}}= & \frac{l}{4 G}\left(\nu^{2}+3\right) \int_{0}^{\Lambda} d r r^{*}(r)-\frac{l}{4 G}\left(\nu^{2}+3\right) \Lambda r_{\Lambda}^{*}+\frac{\left(\nu^{2}+3\right) l}{4 G} r_{h}\left(r_{\Lambda}^{*}-r^{*}(0)\right) \\
& +\frac{l}{2 G} \frac{\left(\nu^{2}+3\right) r_{h}}{\sqrt{3\left(\nu^{2}-1\right)}} \arctan \left(\frac{\sqrt{3\left(\nu^{2}-1\right) \Lambda}}{\sqrt{\left(\nu^{2}+3\right) r_{h}+3\left(\nu^{2}-1\right) \Lambda}}\right) \\
& +\frac{l}{4 G} \sqrt{\Lambda \Psi(\Lambda)} \log \left|\frac{\tilde{L}^{2}}{4 l^{2}} \frac{\left(\nu^{2}+3\right)\left(\Lambda-r_{h}\right)\left[\left(\nu^{2}+3\right) r_{h}+6\left(\nu^{2}-1\right) \Lambda\right]^{2}}{\Lambda \Psi^{2}(\Lambda)}\right| .
\end{aligned}
$$

The divergent parts of the total complexity are:

$$
I^{\mathrm{tot}}=\frac{l}{4 G} \sqrt{3\left(\nu^{2}-1\right)} \Lambda\left(\log \left|\frac{\tilde{L}^{2}}{l^{2}}\left(\nu^{2}+3\right)\right|-1\right)-\frac{l}{8 G} \frac{\nu^{2}+3}{\sqrt{3\left(\nu^{2}-1\right)}} r_{h} \log \Lambda+\mathcal{O}\left(\Lambda^{0}\right) .
$$

This reproduces eq. (3.34) in the $r_{-} \rightarrow 0$ limit.

\section{A.2 External action}

The bulk and the counterterm action can be obtained in the same way as in appendix A.1:

$$
\begin{aligned}
I_{\mathcal{V}}^{\text {out }} & =-\frac{l}{4 G} \sqrt{3\left(\nu^{2}-1\right)} \Lambda-\frac{l}{8 G} \frac{7 \nu^{2}-3}{\sqrt{3\left(\nu^{2}-1\right)}} r_{h} \log \Lambda+\frac{l}{4 G}\left(\nu^{2}+3\right) r_{h} r_{\Lambda}^{*}+\mathcal{O}\left(\Lambda^{0}\right), \\
I_{\mathrm{ct}}^{\text {out }} & =-\frac{l}{4 G} \sqrt{\Lambda \psi(\Lambda)} \log \left|\frac{4 l^{4}}{A^{2} \tilde{L}^{2}} \frac{\Lambda \Psi(\Lambda)}{\left[6\left(\nu^{2}-1\right) \Lambda+\left(\nu^{2}+3\right) r_{h}\right]^{2}}\right|+\mathcal{O}\left(\Lambda^{0}\right) .
\end{aligned}
$$

There is no spacelike or timelike boundary, so there is no contribution from the GHY term.

As in the rotating case, we need to be careful with the regularization of the joints at the horizon; we use again the same method as in [46]. From (3.13) in this situation, we find

$$
\begin{aligned}
I_{\mathcal{J}}^{\text {out }}= & -\frac{l}{4 G} \sqrt{r_{h} \Psi\left(r_{h}\right)}\left[-\log \left|\frac{l^{2}}{A^{2}} \frac{f\left(r_{\epsilon_{U}, \epsilon_{V}}\right)}{2 R\left(r_{h}\right)}\right|+\log \left|\frac{l^{2}}{A^{2}} \frac{f\left(r_{U_{0}, \epsilon_{V}}\right)}{2 R\left(r_{h}\right)}\right|+\log \left|\frac{l^{2}}{A^{2}} \frac{f\left(r_{\epsilon_{U}, V_{0}}\right)}{2 R\left(r_{h}\right)}\right|\right] \\
& +\frac{l}{4 G} \sqrt{\Lambda \Psi(\Lambda)} \log \left|\frac{l^{2}}{A^{2}} \frac{f(\Lambda))}{2 R(\Lambda)}\right| .
\end{aligned}
$$


In this case it is convenient to add and subtract the joint term $\frac{l}{2 G} \nu r_{h} \log \left|\frac{l^{2}}{A^{2}} \frac{f\left(r_{\epsilon_{U}, \epsilon_{V}}\right)}{2 \nu r_{h}}\right|$ and to use the relation (3.38) to get

$$
\begin{aligned}
I_{\mathcal{J}}^{\text {out }}= & -\frac{l}{2 G} \nu r_{h}\left[\log \left(U_{0} V_{0}\right)+\log \left|\frac{l^{2}}{A^{2}} \frac{f\left(r_{\epsilon_{U}, \epsilon_{V}}\right)}{2 \nu r_{h}}\right|-\log \left(\epsilon_{U} \epsilon_{V}\right)\right] \\
& +\frac{l}{4 G} \sqrt{\Lambda \Psi(\Lambda)} \log \left|\frac{l^{2}}{A^{2}} \frac{\left(\nu^{2}+3\right)\left(\Lambda-r_{h}\right)}{\Psi(\Lambda)}\right| .
\end{aligned}
$$

Finally, the expression simplifies by means of eqs. (3.37) and (3.39):

$$
I_{\mathcal{J}}^{\text {out }}=-\frac{l}{2 G}\left[\nu r_{h}\left(\frac{\nu^{2}+3}{2 \nu} r_{\Lambda}^{*}+F\left(r_{h}\right)\right)-\frac{1}{2} \sqrt{\Lambda \Psi(\Lambda)} \log \left|\frac{l^{2}}{A^{2}} \frac{\left(\nu^{2}+3\right)\left(\Lambda-r_{h}\right)}{\Psi(\Lambda)}\right|\right] .
$$

The function $F(r)$, which can be obtained from eq. (3.40), is finite and it is not needed to find the divergence structure. Adding all the terms outside the horizon, we finally obtain

$$
I^{\text {out }}=\frac{l}{4 G} \sqrt{3\left(\nu^{2}-1\right)} \Lambda\left(\log \left|\frac{\tilde{L}^{2}}{l^{2}}\left(\nu^{2}+3\right)\right|-1\right)-\frac{l}{8 G} \frac{7 \nu^{2}-3}{\sqrt{3\left(\nu^{2}-1\right)}} r_{h} \log \Lambda+\mathcal{O}\left(\Lambda^{0}\right) .
$$

This results reproduces eq. (3.44) in the $r_{-} \rightarrow 0$ limit.

\section{A.3 Volume}

The volume is given by the induced metric computed from the non-rotating metric:

$$
V(L)=\int_{0}^{2 \pi} d \theta \int_{r_{h}}^{\Lambda} d r \sqrt{h}=2 \pi l^{2} \int_{r_{h}}^{\Lambda} d r \sqrt{\frac{3\left(\nu^{2}-1\right) r+\left(\nu^{2}+3\right) r_{h}}{4\left(\nu^{2}+3\right)\left(r-r_{h}\right)}} .
$$

We introduce the coordinate $R=r / r_{h}$ and we obtain

$$
V(L)=2 \pi l^{2} r_{h} \int_{1}^{\Lambda / r_{h}} d R \sqrt{\frac{3\left(\nu^{2}-1\right) R+\left(\nu^{2}+3\right)}{4\left(\nu^{2}+3\right)(R-1)}} .
$$

This expression can be analytically solved, giving

$$
\begin{gathered}
V(L)=2 \pi l^{2} r_{h}\left[\sqrt{\frac{\left(\nu^{2}+3\right)+3 R\left(\nu^{2}-1\right)}{\nu^{2}+3} \frac{\sqrt{R-1}}{2}}\right. \\
\left.+\frac{2 \nu^{2} \log \left(\frac{\sqrt{3\left(\nu^{2}-1\right)(R-1)}+\sqrt{3+\nu^{2}+3 R\left(\nu^{2}-1\right)}}{2 \nu}\right)}{\sqrt{3\left(\nu^{2}-1\right)\left(\nu^{2}+3\right)}}\right]_{R=1}^{R=\Lambda / r_{h}} .
\end{gathered}
$$

This gives the following result:

$$
\begin{aligned}
V(L)= & \pi l^{2} \sqrt{\frac{3\left(\nu^{2}-1\right)}{\nu^{2}+3}} \Lambda+\frac{2 \pi l^{2} \nu^{2} r_{h}}{\sqrt{3\left(\nu^{2}-1\right)\left(\nu^{2}+3\right)}} \log \left(\frac{\Lambda}{r_{h}}\right) \\
& +\pi l^{2} r_{h} \frac{\left(3-\nu^{2}\right)+2 \nu^{2} \log \left[\frac{3\left(\nu^{2}-1\right)}{\nu^{2}}\right]}{2 \sqrt{3\left(\nu^{2}-1\right)\left(\nu^{2}+3\right)}}+\mathcal{O}\left(\Lambda^{-1}\right) .
\end{aligned}
$$

The divergent parts of this expression reproduce eq. (4.6) in the $r_{-} \rightarrow 0$ limit. 


\section{B Subsystem complexity and temperature}

Let us compute the temperature dependence of $M$ at constant $J$, which is the specific heat at constant $J$ :

$$
C_{J}=\left.\frac{\partial M}{\partial T}\right|_{J}=\frac{\partial M}{\partial r_{+}} \frac{\partial r_{+}}{\partial T}+\frac{\partial M}{\partial r_{-}} \frac{\partial r_{-}}{\partial T} .
$$

The quantities $\frac{\partial r_{+}}{\partial T}$ and $\frac{\partial r_{-}}{\partial T}$ can be computed from the inverse of the matrix

$$
\left(\begin{array}{ll}
\frac{\partial T}{\partial r_{+}} & \frac{\partial T}{\partial r_{-}} \\
\frac{\partial J}{\partial r_{+}} & \frac{\partial \bar{J}}{\partial r_{-}}
\end{array}\right)
$$

which can be directly calculated from eqs. (2.5) and (2.8). This gives (here $\epsilon=r_{-} / r_{+}$):

$$
C_{J}=\frac{\pi l r_{+}}{4 G} \frac{\nu(\epsilon-1)\left(\epsilon\left(-3 \nu^{2}+2 \nu \sqrt{\left(\nu^{2}+3\right) \epsilon}+3\right)-2 \nu \sqrt{\left(\nu^{2}+3\right) \epsilon}\right)}{\epsilon\left(\nu^{2}(4 \epsilon-1)-3\right)} .
$$

$C_{J}$ is negative for $0<\epsilon<\frac{\nu^{2}+3}{4 \nu^{2}}$ and positive for $\frac{\nu^{2}+3}{4 \nu^{2}}<\epsilon<1$. For $\epsilon=0$ and $\epsilon=\frac{\nu^{2}+3}{4 \nu^{2}}, C_{J}$ is diverging and there is a second order phase transition, similar to the one which occurs for Kerr and Reissner-Nordström black holes in flat spacetime [74].

With a similar method, one can compute the temperature dependence of $K_{+}$and $K_{-}$. The result is:

$$
\left.\frac{\partial K_{+}}{\partial T}\right|_{J}=\frac{\hat{a}}{\hat{b}},\left.\quad \frac{\partial K_{-}}{\partial T}\right|_{J}=\frac{\hat{c}}{\hat{b}}
$$

where

$$
\begin{aligned}
\hat{a}= & 2 \pi l r_{+}\left(\sqrt{\left(\nu^{2}+3\right) r_{+}^{2} \epsilon}-2 \nu r_{+}\right)^{2}\left(\nu\left(\nu^{2}((\epsilon-18) \epsilon-7)+3 \epsilon(\epsilon+6)+3\right)\right. \\
& \left.\times \sqrt{\left(\nu^{2}+3\right) r_{+}^{2} \epsilon}-r_{+} \epsilon\left(-31 \nu^{4}+6 \nu^{2}+\left(\nu^{2}+3\right)^{2} \epsilon+9\right)\right), \\
\hat{b}= & 3\left(\nu^{2}-1\right) \sqrt{\left(\nu^{2}+3\right) r_{+}^{2} \epsilon}\left(4 \nu \sqrt{\left(\nu^{2}+3\right) r_{+}^{2} \epsilon}+\left(\nu^{2}+3\right) r_{+}(-\epsilon-1)\right) \\
& \left(2 \nu(\epsilon+1) \sqrt{\left(\nu^{2}+3\right) r_{+}^{2}} \epsilon-\left(5 \nu^{2}+3\right) r_{+} \epsilon\right) . \\
\hat{c}= & 2 \pi l r_{+}\left(\sqrt{\left(\nu^{2}+3\right) r_{+}^{2} \epsilon}-2 \nu r_{+}\right)^{2}\left(\nu\left(\nu^{2}(\epsilon(7 \epsilon+18)-1)-3(\epsilon(\epsilon+6)+1)\right)\right. \\
& \left.\times \sqrt{\left(\nu^{2}+3\right) r_{+}^{2} \epsilon}+r_{+} \epsilon\left(\left(\nu^{2}+3\right)^{2}+\left(-31 \nu^{4}+6 \nu^{2}+9\right) \epsilon\right)\right) .
\end{aligned}
$$

Open Access. This article is distributed under the terms of the Creative Commons Attribution License (CC-BY 4.0), which permits any use, distribution and reproduction in any medium, provided the original author(s) and source are credited.

\section{References}

[1] J.M. Maldacena, Eternal black holes in Anti-de Sitter, JHEP 04 (2003) 021 [hep-th/0106112] [INSPIRE]. 
[2] T. Hartman and J. Maldacena, Time evolution of entanglement entropy from black hole interiors, JHEP 05 (2013) 014 [arXiv: 1303.1080] [INSPIRE].

[3] L. Susskind, Computational complexity and black hole horizons, Fortsch. Phys. 64 (2016) 44 [arXiv: 1403.5695] [INSPIRE].

[4] D. Stanford and L. Susskind, Complexity and shock wave geometries, Phys. Rev. D 90 (2014) 126007 [arXiv:1406.2678] [INSPIRE].

[5] L. Susskind, Entanglement is not enough, Fortsch. Phys. 64 (2016) 49 [arXiv:1411.0690] [INSPIRE].

[6] M.A. Nielsen, A geometric approach to quantum circuit lower bounds, Quant. Inf. Comput. 6 (2006) 213 [quant-ph/0502070].

[7] M.R. Dowling and M.A. Nielsen, The geometry of quantum computation, Quant. Inf. Comput. 8 (2008) 861 [quant-ph/0701004].

[8] R. Jefferson and R.C. Myers, Circuit complexity in quantum field theory, JHEP 10 (2017) 107 [arXiv: 1707.08570] [INSPIRE].

[9] S. Chapman, M.P. Heller, H. Marrochio and F. Pastawski, Toward a definition of complexity for quantum field theory states, Phys. Rev. Lett. 120 (2018) 121602 [arXiv:1707.08582] [INSPIRE].

[10] K. Hashimoto, N. Iizuka and S. Sugishita, Time evolution of complexity in Abelian gauge theories, Phys. Rev. D 96 (2017) 126001 [arXiv:1707.03840] [INSPIRE].

[11] R.-Q. Yang, C. Niu, C.-Y. Zhang and K.-Y. Kim, Comparison of holographic and field theoretic complexities for time dependent thermofield double states, JHEP 02 (2018) 082 [arXiv: 1710.00600] [INSPIRE].

[12] R. Khan, C. Krishnan and S. Sharma, Circuit complexity in fermionic field theory, Phys. Rev. D 98 (2018) 126001 [arXiv:1801.07620] [INSPIRE].

[13] L. Hackl and R.C. Myers, Circuit complexity for free fermions, JHEP 07 (2018) 139 [arXiv: 1803.10638] [INSPIRE].

[14] S. Chapman et al., Complexity and entanglement for thermofield double states, SciPost Phys. 6 (2019) 034 [arXiv: 1810.05151] [INSPIRE].

[15] P. Caputa et al., Liouville action as path-integral complexity: from continuous tensor networks to AdS/CFT, JHEP 11 (2017) 097 [arXiv:1706. 07056] [INSPIRE].

[16] A. Bhattacharyya et al., Path-integral complexity for perturbed CFTs, JHEP 07 (2018) 086 [arXiv: 1804.01999] [INSPIRE].

[17] A.R. Brown et al., Holographic complexity equals bulk action?, Phys. Rev. Lett. 116 (2016) 191301 [arXiv: 1509.07876] [INSPIRE].

[18] A.R. Brown et al., Complexity, action and black holes, Phys. Rev. D 93 (2016) 086006 [arXiv: 1512.04993] [INSPIRE].

[19] G. Hayward, Gravitational action for space-times with nonsmooth boundaries, Phys. Rev. D 47 (1993) 3275 [INSPIRE].

[20] L. Lehner, R.C. Myers, E. Poisson and R.D. Sorkin, Gravitational action with null boundaries, Phys. Rev. D 94 (2016) 084046 [arXiv:1609.00207] [INSPIRE]. 
[21] J. Couch, W. Fischler and P.H. Nguyen, Noether charge, black hole volume and complexity, JHEP 03 (2017) 119 [arXiv: 1610.02038] [INSPIRE].

[22] D. Carmi et al., On the time dependence of holographic complexity, JHEP 11 (2017) 188 [arXiv: 1709.10184] [INSPIRE].

[23] R.-Q. Yang et al., Principles and symmetries of complexity in quantum field theory, Eur. Phys. J. C 79 (2019) 109 [arXiv: 1803.01797] [INSPIRE].

[24] K. Hashimoto, N. Iizuka and S. Sugishita, Thoughts on holographic complexity and its basis-dependence, Phys. Rev. D 98 (2018) 046002 [arXiv:1805.04226] [INSPIRE].

[25] S. Chapman, H. Marrochio and R.C. Myers, Complexity of formation in holography, JHEP 01 (2017) 062 [arXiv: 1610.08063] [INSPIRE].

[26] R.-G. Cai et al., Action growth for AdS black holes, JHEP 09 (2016) 161 [arXiv: 1606. 08307] [INSPIRE].

[27] A. Reynolds and S.F. Ross, Divergences in holographic complexity, Class. Quant. Grav. 34 (2017) 105004 [arXiv:1612.05439] [INSPIRE].

[28] M. Moosa, Evolution of complexity following a global quench, JHEP 03 (2018) 031 [arXiv: 1711.02668] [INSPIRE].

[29] M. Moosa, Divergences in the rate of complexification, Phys. Rev. D 97 (2018) 106016 [arXiv: 1712.07137] [INSPIRE].

[30] S. Chapman, H. Marrochio and R.C. Myers, Holographic complexity in Vaidya spacetimes. Part I, JHEP 06 (2018) 046 [arXiv: 1804.07410] [INSPIRE].

[31] S. Chapman, H. Marrochio and R.C. Myers, Holographic complexity in Vaidya spacetimes. Part II, JHEP 06 (2018) 114 [arXiv:1805.07262] [INSPIRE].

[32] J.L.F. Barbon and E. Rabinovici, Holographic complexity and spacetime singularities, JHEP 01 (2016) 084 [arXiv: 1509.09291] [INSPIRE].

[33] S. Bolognesi, E. Rabinovici and S.R. Roy, On some universal features of the holographic quantum complexity of bulk singularities, JHEP 06 (2018) 016 [arXiv:1802.02045] [INSPIRE].

[34] B. Swingle and Y. Wang, Holographic complexity of Einstein-Maxwell-dilaton gravity, JHEP 09 (2018) 106 [arXiv:1712.09826] [INSPIRE].

[35] Y.-S. An and R.-H. Peng, Effect of the dilaton on holographic complexity growth, Phys. Rev. D 97 (2018) 066022 [arXiv:1801.03638] [INSPIRE].

[36] M. Headrick, V.E. Hubeny, A. Lawrence and M. Rangamani, Causality 83 holographic entanglement entropy, JHEP 12 (2014) 162 [arXiv:1408.6300] [INSPIRE].

[37] M. Alishahiha, Holographic complexity, Phys. Rev. D 92 (2015) 126009 [arXiv:1509.06614] [INSPIRE].

[38] S. Ryu and T. Takayanagi, Holographic derivation of entanglement entropy from AdS/CFT, Phys. Rev. Lett. 96 (2006) 181602 [hep-th/0603001] [InSPIRE].

[39] D. Carmi, R.C. Myers and P. Rath, Comments on holographic complexity, JHEP 03 (2017) 118 [arXiv: 1612.00433] [INSPIRE].

[40] O. Ben-Ami and D. Carmi, On volumes of subregions in holography and complexity, JHEP 11 (2016) 129 [arXiv:1609.02514] [INSPIRE]. 
[41] R. Abt et al., Topological complexity in AdS $S_{3} / C F T_{2}$, Fortsch. Phys. 66 (2018) 1800034 [arXiv: 1710.01327] [INSPIRE].

[42] R. Abt et al., Holographic subregion complexity from kinematic space, JHEP 01 (2019) 012 [arXiv: 1805.10298] [INSPIRE].

[43] P. Roy and T. Sarkar, Note on subregion holographic complexity, Phys. Rev. D 96 (2017) 026022 [arXiv: 1701.05489] [INSPIRE].

[44] P. Roy and T. Sarkar, Subregion holographic complexity and renormalization group flows, Phys. Rev. D 97 (2018) 086018 [arXiv:1708.05313] [INSPIRE].

[45] A. Bhattacharya, K.T. Grosvenor and S. Roy, Higher-order corrections to holographic entanglement entropy and subregion complexity in the AdS black hole background, arXiv: 1905.02220 [INSPIRE].

[46] C.A. Agón, M. Headrick and B. Swingle, Subsystem complexity and holography, JHEP 02 (2019) 145 [arXiv: 1804.01561] [INSPIRE].

[47] M. Alishahiha, K. Babaei Velni and M.R. Mohammadi Mozaffar, Black hole subregion action and complexity, Phys. Rev. D 99 (2019) 126016 [arXiv:1809.06031] [InSPIRE].

[48] E. Cáceres, J. Couch, S. Eccles and W. Fischler, Holographic purification complexity, Phys. Rev. D 99 (2019) 086016 [arXiv: 1811.10650] [inSPIRE].

[49] M. Ghodrati et al., The connection between holographic entanglement and complexity of purification, JHEP 09 (2019) 009 [arXiv: 1902.02475] [INSPIRE].

[50] D. Anninos et al., Warped AdS 3 Black Holes, JHEP 03 (2009) 130 [arXiv:0807.3040] [INSPIRE].

[51] D. Anninos, Hopfing and puffing warped Anti-de Sitter space, JHEP 09 (2009) 075 [arXiv:0809.2433] [INSPIRE].

[52] S. Detournay, T. Hartman and D.M. Hofman, Warped conformal field theory, Phys. Rev. D 86 (2012) 124018 [arXiv: 1210.0539] [INSPIRE].

[53] D.M. Hofman and B. Rollier, Warped conformal field theory as lower spin gravity, Nucl. Phys. B 897 (2015) 1 [arXiv:1411.0672] [InSPIRE].

[54] K. Jensen, Locality and anomalies in warped conformal field theory, JHEP 12 (2017) 111 [arXiv:1710.11626] [INSPIRE].

[55] D. Anninos, J. Samani and E. Shaghoulian, Warped entanglement entropy, JHEP 02 (2014) 118 [arXiv: 1309.2579] [INSPIRE].

[56] A. Castro, D.M. Hofman and N. Iqbal, Entanglement entropy in warped conformal field theories, JHEP 02 (2016) 033 [arXiv:1511.00707] [INSPIRE].

[57] T. Azeyanagi, S. Detournay and M. Riegler, Warped black holes in lower-spin gravity, Phys. Rev. D 99 (2019) 026013 [arXiv:1801.07263] [INSPIRE].

[58] W. Song, Q. Wen and J. Xu, Generalized gravitational entropy for warped Anti-de Sitter space, Phys. Rev. Lett. 117 (2016) 011602 [arXiv:1601.02634] [INSPIRE].

[59] W. Song, Q. Wen and J. Xu, Modifications to holographic entanglement entropy in warped CFT, JHEP 02 (2017) 067 [arXiv:1610.00727] [INSPIRE].

[60] M. Ghodrati, Complexity growth in massive gravity theories, the effects of chirality and more, Phys. Rev. D 96 (2017) 106020 [arXiv:1708.07981] [INSPIRE]. 
[61] R. Auzzi, S. Baiguera and G. Nardelli, Volume and complexity for warped AdS black holes, JHEP 06 (2018) 063 [arXiv: 1804.07521] [INSPIRE].

[62] R. Auzzi et al., Complexity and action for warped AdS black holes, JHEP 09 (2018) 013 [arXiv: 1806.06216] [INSPIRE].

[63] H. Dimov, R.C. Rashkov and T. Vetsov, Thermodynamic information geometry and complexity growth of a warped AdS black hole and the warped $A d S_{3} / C F T_{2}$ correspondence, Phys. Rev. D 99 (2019) 126007 [arXiv:1902.02433] [InSPIRE].

[64] K.A. Moussa, G. Clement and C. Leygnac, The black holes of topologically massive gravity, Class. Quant. Grav. 20 (2003) L277 [gr-qc/0303042] [INSPIRE].

[65] A. Bouchareb and G. Clement, Black hole mass and angular momentum in topologically massive gravity, Class. Quant. Grav. 24 (2007) 5581 [arXiv:0706.0263] [InSPIRE].

[66] M. Bañados, C. Teitelboim and J. Zanelli, The Black hole in three-dimensional space-time, Phys. Rev. Lett. 69 (1992) 1849 [hep-th/9204099] [INSPIRE].

[67] M. Bañados, M. Henneaux, C. Teitelboim and J. Zanelli, Geometry of the $(2+1)$ black hole, Phys. Rev. D 48 (1993) 1506 [Erratum ibid. D 88 (2013) 069902] [gr-qc/9302012] [INSPIRE].

[68] M. Bañados, G. Barnich, G. Compere and A. Gomberoff, Three dimensional origin of Godel spacetimes and black holes, Phys. Rev. D 73 (2006) 044006 [hep-th/0512105] [INSPIRE].

[69] G. Barnich and G. Compere, Conserved charges and thermodynamics of the spinning Godel black hole, Phys. Rev. Lett. 95 (2005) 031302 [hep-th/0501102] [INSPIRE].

[70] G. Clement, Warped AdS $S_{3}$ black holes in new massive gravity, Class. Quant. Grav. 26 (2009) 105015 [arXiv:0902 .4634] [INSPIRE].

[71] F. Jugeau, G. Moutsopoulos and P. Ritter, From accelerating and Poincaré coordinates to black holes in spacelike warped $A d S_{3}$ and back, Class. Quant. Grav. 28 (2011) 035001 [arXiv: 1007.1961] [INSPIRE].

[72] R.-Q. Yang, C. Niu and K.-Y. Kim, Surface counterterms and regularized holographic complexity, JHEP 09 (2017) 042 [arXiv:1701.03706] [INSPIRE].

[73] A. Akhavan and F. Omidi, On the Role of Counterterms in Holographic Complexity, arXiv: 1906.09561 [INSPIRE].

[74] P.C.W. Davies, Thermodynamics of black holes, Proc. Roy. Soc. Lond. A 353 (1977) 499. 